\title{
ENTROPY THEORY FROM ORBITAL POINT OF VIEW
}

\author{
Alexandre I. Danilenko
}

\begin{abstract}
Inspired by [RW], we develop an orbital approach to the entropy theory for actions of countable amenable groups. This is applied to extend-with new short proofs - the recent results about uniform mixing of actions with completely positive entropy [RW], Pinsker factors and the relative disjointness problems [GTW], Abramov-Rokhlin entropy addition formula [ZW], etc. Unlike the cited papers our work is independent of the standard machinery developed by Ornstein-Weiss [OW] or Kieffer [Ki]. We do not use non-orbital tools like Rokhlin lemma, Shannon-McMillan theorem, castle analysis, joining techniques for amenable actions, etc. which play an essential role in $[\mathrm{RW}],[\mathrm{ZW}]$ and [GTW].
\end{abstract}

\section{INTRODUCTION}

The classical entropy theory was developed for measure preserving transformations i.e. $\mathbb{Z}$-actions. Afterwards it became clear that a part of this theory can be lifted to actions of countable amenable groups. For this purpose Ornstein and Weiss worked out in $[\mathrm{OW}]$ a fundamental machinery based on the combinatorial analysis for such groups (see also [Ki] and [WZ] for an alternative approach). They proved in particular Rokhlin lemma, Shannon-McMillan theorem, isomorphism theorem for Bernoullian actions of amenable groups, etc. The principal obstacle for extending other results of the classical entropy theory for $\mathbb{Z}$-actions to general amenable actions is lack of a good analogue for the past-algebra of a process because of there is no a natural "time" order on an amenable group. Thus a problem is to develop an entropy theory without past. Glasner, Thouvenot and Weiss succeeded this partially in a recent paper [GTW] on the Pinsker algebras of amenable dynamical systems. To this end they used the basic machinery from $[\mathrm{OW}]$ and a techniques related to joinings. Another progress was achieved by Rudolph and Weiss in [RW] where they proved that the actions with completely positive entropy (CPE) are uniformly mixing. Their exposition is also based heavily on [OW] and - rather surprisingly in this context - on the orbit theory for amenable actions. Being intrigued by the latter we try to understand better the significance of the orbit theory in their theorem and in the entropy theory in general.

As it turns out it is possible to develop a purely orbital approach to the entropy theory for amenable actions which is independent of $[\mathrm{OW}]$ and $[\mathrm{Ki}]$. This is the goal of the present work.

In what follows we provide an informal outline of our paper. The main body of it consists of two parts. The first one $(\S 2)$ is more abstract. The objects considered

1991 Mathematics Subject Classification. Primary 37A35, 37A20; Secondary 37A15.

Key words and phrases. Discrete equivalence relation, cocycle, amenable group, entropy.

Typeset by $\mathcal{A}_{\mathcal{M S}}-\mathrm{T}_{\mathrm{EX}}$ 
here are of orbital nature: a measure preserving discrete equivalence relation $\mathcal{R}$ and a cocycle $\alpha$ of it with values in the transformation group of a Lebesque space. For a finite partition $P$, we define an entropy $h(\alpha, P)$ of the "process" $(\alpha, P)$ (see Definition 2.4). The entropy and the Pinsker algebra of $\alpha$ are now determined in a natural way: $h(\alpha):=\sup _{P} h(\alpha, P)$ and $\Pi(\alpha):=\vee\{P \mid h(\alpha, P)=0\}$ (Definitions 2.4, 2.5). If $\alpha$ is hyperfinite, i.e. there exists a filtration $\left(\mathcal{R}_{n}\right)_{n \geq 1}$ of $\mathcal{R}$ by type I subrelations, then $h(\alpha, P)=\lim _{n \rightarrow \infty} h\left(\alpha \uparrow \mathcal{R}_{n}, P\right)$ (Corollary 2.7). As usual, the sign $\uparrow$ stands for the "restriction". This approximation result is constantly used in our work. Next, we demonstrate that the Pinsker algebra of $\alpha$ is invariant under the $\alpha$-skew product extension of the "symmetry group" of $\alpha$ (Corollary 2.11). Moreover, if $\alpha$ is "sufficiently symmetric" then $\Pi(\alpha)$ splits into the product of the entire base $\sigma$-algebra and a sub- $\sigma$-algebra in the fiber (Theorem 2.12). For $\alpha$ recurrent (in $\mathrm{K}$. Schmidt terminology $[\mathrm{Sc}]), \Pi(\alpha)$ is the largest possible which is equivalent to $h(\alpha)=0$ (Theorem 2.13). Next, we use the "measured" index theory of FeldmanSutherland-Zimmer [FSZ] to show that given a nested pair of ergodic hyperfinite subrelations of finite index, then $h(\alpha\lceil\mathcal{S})=\operatorname{ind}(\mathcal{R}: \mathcal{S}) \cdot h(\mathcal{R})$ (Theorem 2.16).

The second part of the paper (Sections 3-6) is devoted to applications of the results of $\S 2$ to amenable group actions. We first define a "virtual" entropy of a process $(T, P)$ consisting of an action $T$ of a countable amenable group and a finite partition $P$. This is $h\left(\alpha^{\prime}, P^{\prime}\right)$ for a cocycle $\alpha^{\prime}$ of a discrete equivalence relation and a partition $P^{\prime}$ which are associated to $(T, P)$ in some special way (Definition 3.1 ). We then show that the virtual entropy equals to the entropy introduced in [Ki] and [OW] (Theorem 3.3). Being combined with the following two fundamental theorems of the orbit theory:

$(\bullet)$ the orbit equivalence relation of a measure preserving action of a countable amenable group is generated by a single transformation [CFW],

$(\bullet)$ any two ergodic measure preserving transformations are orbit equivalent $[\mathrm{Dy}]$,

the virtual entropy fits well to transfer many of the results of the classical ergodic theory to general amenable actions. We realize this transfer by means of Corollaries 3.4, 3.7 and Theorem 3.6 and do not use Følner sequences and Rokhlin lemma for amenable actions anywhere. It is worthwhile to remark that $[\mathrm{CFW}]$ avoids the use of Rokhlin lemma as well. Thus our approach to the entropy theory for amenable group actions is completely independent of [OW].

We reprove and extend the main results of [RW], [GTW] and [WZ] eliminating from their proofs the "non-orbital" tools like Rokhlin lemma, ergodic theorems, Shannon-McMillan theorem, castle analysis, joining techniques, etc. Since we replace them by more "symmetric", "non-coordinate" orbital techniques, this leads to shorter proofs. We list these applications as follows (see $\S 2$ for the definitions of the relative entropy and the Pinsker algebra).

Theorem 0.1. Let $T=\left\{T_{g}\right\}_{g \in G}$ be a measure preserving action of a countable amenable group $G$ on a standard probability space $\left(Y, \mathfrak{B}_{Y}, \nu\right)$ and $\mathfrak{E} \subset \mathfrak{B}_{Y}$ a factor of $T$. Suppose that $T$ is $\mathfrak{E}$-relatively CPE. Then given a finite partition $Q$ of $Y$ and $\epsilon>0$, there is a finite subset $K \subset G$ such that

$$
\left|\frac{1}{\# F} H\left(\bigvee_{g \in F} T_{g}^{-1} Q \mid \mathfrak{E}\right)-H(Q \mid \mathfrak{E})\right|<\epsilon
$$

for any finite subset $F \subset G$ with $g_{1} g_{2}^{-1} \notin K$ for all $g_{1} \neq g_{2} \in F$. 
Theorem 0.2. Let $\mathfrak{E}$ be a factor of a $G$-action $T$. Then $h(T)=h(T\lceil\mathfrak{E})+h(T \mid \mathfrak{E})$, where the first term denotes the entropy of the factor-action and the second term denotes the $\mathfrak{E}$-relative entropy.

Theorem 0.3. Let $T$ and $U$ be free actions of countable amenable groups $G$ and $F$ respectively on $\left(Y, \mathfrak{B}_{Y}, \nu\right)$ and $\mathfrak{E}$ a class-bijective factor of each of these actions. Suppose that $T$ and $U$ are $\mathfrak{E}$-orbit equivalent, i.e. they have the same orbits and for each $g \in G$ and $f \in F$, the subset $\left\{y \in Y \mid T_{g} y=U_{f} y\right\}$ is $\mathfrak{E}$-measurable. Then for each finite partition $Q$ of $Y$ we have $h(T, Q \mid \mathfrak{E})=h(U, Q \mid \mathfrak{E})$.

Theorem 0.4. Let $\mathfrak{A}_{1}, \mathfrak{A}_{2}$, $\mathfrak{E}$ be three factors of a $G$-action $T$ with $\mathfrak{E} \subset \mathfrak{A}_{1} \cap \mathfrak{A}_{2}$.

(i) If $T\left\lceil\mathfrak{A}_{1}\right.$ is $\mathfrak{E}$-relatively $C P E$ and $h\left(\left(T\left\lceil\mathfrak{A}_{2}\right) \mid \mathfrak{E}\right)=0\right.$ then $\mathfrak{A}_{1}$ and $\mathfrak{A}_{2}$ are $\mathfrak{E}$-relatively independent.

(ii) If $T\left\lceil\mathfrak{A}_{1}\right.$ is $\mathfrak{E}$-relatively $C P E$ then $T\left\lceil\left(\mathfrak{A}_{1} \vee \mathfrak{A}_{2}\right)\right.$ is $\mathfrak{A}_{2}$-relatively $C P E$.

(iii) If $\mathfrak{A}_{1}$ and $\mathfrak{A}_{2}$ are $\mathfrak{E}$-relatively independent then $\Pi\left(\left(T \uparrow\left(\mathfrak{A}_{1} \vee \mathfrak{A}_{2}\right)\right) \mid \mathfrak{E}\right)=$ $\Pi\left(\left(T\left\lceil\mathfrak{A}_{1}\right) \mid \mathfrak{E}\right) \vee \Pi\left(\left(T\left\lceil\mathfrak{A}_{2}\right) \mid \mathfrak{E}\right)\right.\right.$.

(iv) $\mathfrak{A}_{1}$ and $\mathfrak{A}_{2}$ are $\mathfrak{E}$-relatively independent if and only if the $\mathfrak{E}$-relative Pinsker algebras $\Pi\left(\left(T\left\lceil\mathfrak{A}_{1}\right) \mid \mathfrak{E}\right)\right.$ and $\Pi\left(\left(T\left\lceil\mathfrak{A}_{2}\right) \mid \mathfrak{E}\right)\right.$ are $\mathfrak{E}$-relatively independent and

$$
h\left(\left(T \uparrow\left(\mathfrak{A}_{1} \vee \mathfrak{A}_{2}\right)\right) \mid \mathfrak{E}\right)=h\left(\left(T\left\lceil\mathfrak{A}_{1}\right) \mid \mathfrak{E}\right)+h\left(\left(T \nmid \mathfrak{A}_{2}\right) \mid \mathfrak{E}\right) .\right.
$$

Remark that Theorem 0.1 extends the main result of [RW], where it was assumed additionally that $T$ is free ergodic and $\mathfrak{E}$ is trivial. Theorem 0.2 is the AbramovRokhlin entropy addition formula for amenable dynamical systems i.e. the main result of [WZ]. Originally Theorem 0.3 was proved in [RW] in a different way as an auxiliary statement for their version of Theorem 0.1 . Theorem 0.4 extends the main results of [GTW], where it was assumed that the actions are ergodic. Moreover, (ii) was proved in $[\mathrm{GTW}]$ under an additional condition that $\mathfrak{A}_{1}$ and $\mathfrak{A}_{2}$ are $\mathfrak{E}$-relatively independent. As concern to (iv), only the part "if" of this claim was demonstrated there.

The proof of Theorem 0.1 occupies the final part of Section 3. Sections 4-6 devoted entirely to the proofs of Theorems $0.2-0.4$ respectively. A background material is contained in Section 1.

I would like to thank J.-P. Thouvenot for pointing out a gap in an earlier statement of Theorem 0.4. Originally Theorem 2.13 and Corollary 5.3 were proved in this paper for regular cocycles only. I thank M. Lemańczyk for his advice to extend them for arbitrary recurrent cocycles.

\section{Notation. Preliminaries}

Let $\left(X, \mathfrak{B}_{X}, \mu\right)$ be a standard probability space. Throughout this paper we do not distinguish the objects (like subsets, maps, partitions, etc.) which agree on a $\mu$-conull subset. The trivial sub- $\sigma$-algebra of $\mathfrak{B}_{X}$ is denoted by $\mathfrak{N}_{X}$. Let $\mathfrak{A}_{1}, \mathfrak{A}_{2}$ and $\mathfrak{F}$ be three sub- $\sigma$-algebra of $\mathfrak{B}_{X}$ and $\mu=\int \mu_{x} d(\mu\lceil\mathfrak{F})(x)$ the disintegration of $\mu$ over $\mu \nmid \mathfrak{F}$. We say that $\mathfrak{A}_{1}$ and $\mathfrak{A}_{2}$ are $\mathfrak{F}$-relatively independent if

$$
\mu_{x}\left(A_{1} \cap A_{2}\right)=\mu_{x}\left(A_{1}\right) \mu_{x}\left(A_{2}\right) \quad \text { at }(\mu\lceil\mathfrak{F}) \text {-a.e. } \mathbf{x}
$$

for all subsets $A_{1} \in \mathfrak{A}_{1}$ and $A_{2} \in \mathfrak{A}_{2}$. Clearly, this implies $\mathfrak{A}_{1} \cap \mathfrak{A}_{2} \subset \mathfrak{F}$. The inclusion can be strict: any two subalgebras $\mathfrak{A}_{1}^{\prime} \subset \mathfrak{A}_{1}$ and $\mathfrak{A}_{2}^{\prime} \subset \mathfrak{A}_{2}$ are also $\mathfrak{F}^{-}$ relatively independent. 
Let $\operatorname{Aut}(X, \mu)$ stand for the group of $\mu$-preserving invertible transformations of $X$. We endow it with the (Polish) weak topology, i.e. the weakest topology which makes continuous the following unitary representation:

$$
\operatorname{Aut}(X, \mu) \ni \gamma \mapsto U_{\gamma} \in \mathcal{U}\left(L^{2}(X, \mu)\right)
$$

where $U_{\gamma} f=f \circ \gamma^{-1}$ and the unitary group $\mathcal{U}\left(L^{2}(X, \mu)\right)$ is furnished with the (Polish) strong operator topology. For a sub- $\sigma$-algebra $\mathfrak{E}$ of $\mathfrak{B}_{X}$, denote by $\operatorname{Aut}_{\mathfrak{E}}(X, \mu)$ the sub-collection of automorphisms which preserve $\mathfrak{E}$ invariant. Clearly, $\operatorname{Aut}_{\mathfrak{E}}(X, \mu)$ is a closed subgroup in $\operatorname{Aut}(X, \mu)$.

Orbital background (see [FM, Sc, GoS]). Let a Borel subset $\mathcal{R} \subset X \times X$ be an equivalence relation. For $x \in X$, we denote by $\mathcal{R}(x)$ the $\mathcal{R}$-equivalence class of $x$. Following [FM], we say that $\mathcal{R}$ is discrete if $\# \mathcal{R}(x) \leq \# \mathbb{Z}$ a.e.. $\mathcal{R}$ is measure preserving if it is generated by a countable subgroup $G \subset \operatorname{Aut}(X, \mu)$. This generating subgroup is highly non-unique. $\mathcal{R}$ is of type I if $\# \mathcal{R}(x)<\infty$ a.e. or, equivalently, there is a subset $B \in \mathfrak{B}_{X}$ with $\#(B \cap \mathcal{R}(x))=1$ a.e. Such $B$ is called an $\mathcal{R}$-fundamental domain. We say that $\mathcal{R}$ is countable if $\# \mathcal{R}(x)=\infty$ a.e.. Notice that $\mathcal{R}$ (which is measure preserving) is countable if and only if it is conservative, i.e. $\mathcal{R} \cap(B \times B) \backslash \mathcal{D} \neq \emptyset$ for every $B \in \mathfrak{B}$ of positive measure, where $\mathcal{D}$ stands for the diagonal equivalence relation on $X$. $\mathcal{R}$ is hyperfinite if there exists a sequence $\mathcal{R}_{1} \subset \mathcal{R}_{2} \subset \ldots$ of type $I$ subrelations of $\mathcal{R}$ with $\bigcup_{n} \mathcal{R}_{n}=\mathcal{R}$. The sequence $\left(\mathcal{R}_{n}\right)_{n}$ is called a filtration of $\mathcal{R}$. It follows from [Dy] that a measure preserving discrete equivalence relation is hyperfinite if and only if it is generated by a single transformation. The orbit equivalence relation of a measure preserving action of a countable amenable group is hyperfinite [ $\mathrm{Zi}, \mathrm{CFW}$. $\mathcal{R}$ is ergodic if every $\mathcal{R}$-invariant subset belongs to $\mathfrak{N}_{X}$. Any two ergodic hyperfinite measure preserving countable equivalence relations are isomorphic in the natural sense (i.e. there exists an isomorphism between the measure spaces which intertwines the corresponding equivalence classes) [Dy].

Everywhere below $\mathcal{R}$ is a measure preserving discrete equivalence relation on $\left(X, \mathfrak{B}_{X}, \mu\right)$. We let

$$
\begin{aligned}
{[\mathcal{R}] } & :=\{\gamma \in \operatorname{Aut}(X, \mu) \mid(x, \gamma x) \in \mathcal{R} \text { a.e. }\} \\
N[\mathcal{R}] & :=\{\theta \in \operatorname{Aut}(X, \mu) \mid \theta \mathcal{R}(x)=\mathcal{R}(\theta x) \text { a.e. }\}
\end{aligned}
$$

for the full group of $\mathcal{R}$ and its normalizer respectively.

Let $A$ be a Polish group. A Borel map $\alpha: \mathcal{R} \rightarrow A$ is called a cocycle if

$$
\alpha\left(x, x^{\prime \prime}\right)=\alpha\left(x, x^{\prime}\right) \alpha\left(x, x^{\prime \prime}\right) \quad \text { for all }\left(x, x^{\prime}\right),\left(x^{\prime}, x^{\prime \prime}\right) \in \mathcal{R} .
$$

Two cocycles $\alpha, \beta: \mathcal{R} \rightarrow A$ are cohomologous if there is a Borel map $\phi: X \rightarrow A$ with

$$
\alpha\left(x, x^{\prime}\right)=\phi(x) \beta\left(x, x^{\prime}\right) \phi\left(x^{\prime}\right)^{-1} \quad \text { for all }\left(x, x^{\prime}\right) \in \mathcal{R} \cap B \times B,
$$

where $B$ is a $\mu$-conull subset. We write $\alpha \approx_{\phi} \beta$.

For a transformation $\theta \in N[\mathcal{R}]$, we define a cocycle $\alpha \circ \theta$ by setting

$$
\alpha \circ \theta\left(x, x^{\prime}\right)=\alpha\left(\theta x, \theta x^{\prime}\right) .
$$


Two cocycles $\alpha, \beta: \mathcal{R} \rightarrow A$ are weakly equivalent if $\alpha$ is cohomologous to $\beta \circ \theta$ for a transformation $\theta \in N[\mathcal{R}]$. Clearly, the cohomology and the weak equivalence are equivalence relations on the set of $A$-valued cocycles of $\mathcal{R}$.

A cocycle $\alpha: \mathcal{R} \rightarrow A$ is recurrent if for each neighborhood $U$ of the identity $1_{A}$ in $A$ and a subset $B \in \mathfrak{B}$ of positive measure there exist a subset $B_{1} \in \mathfrak{B}$ and a transformation $\gamma \in[\mathcal{R}]$ such that the following properties are satisfied: $\mu\left(B_{1}\right)>0$, $B_{1} \cup \gamma B_{1} \subset B, \gamma x \neq x$ and $\alpha(x, \gamma x) \in U$ for all $x \in B_{1}$. Notice that if $\alpha$ is recurrent then $\mathcal{R}$ is conservative. Moreover, if $\mathcal{R}$ is conservative then every cocycle of $\mathcal{R}$ with values in a compact group is recurrent. One can check easily that the recurrence is an invariant for the cohomology and the weak equivalence.

Let $\left(Y, \mathfrak{B}_{Y}, \nu\right)$ be another standard probability space and $A$ embedded continuously in $\operatorname{Aut}(Y, \nu)$. Given a cocycle $\alpha: \mathcal{R} \rightarrow A$, we associate a measure preserving discrete equivalence relation $\mathcal{R}(\alpha)$ on $(X \times Y, \mu \times \nu)$ by setting $(x, y) \sim_{\mathcal{R}(\alpha)}\left(x^{\prime}, y^{\prime}\right)$ if $\left(x, x^{\prime}\right) \in \mathcal{R}$ and $y^{\prime}=\alpha\left(x^{\prime}, x\right) y$. Then a one-to-one group homomorphism $[R] \ni$ $\gamma \mapsto \gamma_{\alpha} \in[\mathcal{R}(\alpha)]$ is well defined via the formula

$$
\gamma_{\alpha}(x, y)=(\gamma x, \alpha(\gamma x, x) y),(x, y) \in X \times Y \text {. }
$$

The transformation $\gamma_{\alpha}$ is called the $\alpha$-skew product extension of $\gamma$. The equivalence relation $\mathcal{R}(\alpha)$ is called the $\alpha$-skew product extension of $\mathcal{R}$.

Entropic concepts (see $[\mathrm{Ki}, \mathrm{Ol}, \mathrm{OW}]$ ). Let $G$ be a countable amenable group. Denote by $\operatorname{Fin}(G)$ the set of finite $G$-subsets. Given $K \in \operatorname{Fin}(G)$ and $\epsilon>0$, a subset $F \in \operatorname{Fin}(G)$ is called $[K, \epsilon]$-invariant if

$$
\#\{g \in F \mid K g \subset F\}>(1-\epsilon) \# F
$$

Let $\Phi[K, \epsilon]$ stand for the collection of $[K, \epsilon]$-invariant subsets. Since $G$ is amenable, the collection is non-empty. Moreover,

$$
\Phi\left[K_{1}, \epsilon_{1}\right] \cap \Phi\left[K_{2}, \epsilon_{2}\right] \supset \Phi\left[K_{1} \cup K_{2}, \min \left(\epsilon_{1}, \epsilon_{2}\right)\right] .
$$

Hence the family $\{\Phi[K, \epsilon] \mid K \in \operatorname{Fin}(G), \epsilon>0\}$ is a base of a filter $\Phi$, which is called the amening filter on $G$.

Let $T=\left\{T_{g}\right\}_{g \in G}$ be a free ergodic measure preserving action of $G$ on $\left(Y, \mathfrak{B}_{Y}, \nu\right)$ and $Q$ a finite partition of $Y$. A $T$-invariant sub- $\sigma$-algebra $\mathfrak{E}$ of $\mathfrak{B}_{Y}$ is called a factor of $T$. The restriction of $T$ to $(\mathfrak{E}, \nu \uparrow \mathfrak{E})$ will be denoted by $T \uparrow \mathfrak{E}$.

The $\mathfrak{E}$-relative entropy of the process $(T, Q)$ is

$$
h(T, Q \mid \mathfrak{E}):=\inf \left\{\frac{1}{\# F} H\left(\bigvee_{g \in F} T_{g}^{-1} Q \mid \mathfrak{E}\right) \mid F \in \operatorname{Fin}(G)\right\}
$$

Theorem 1.1 (cf. [Ol, RW]). $h(T, Q \mid \mathfrak{E})=\lim _{\Phi} \frac{1}{\# F} H\left(\bigvee_{g \in F} T_{g}^{-1} Q \mid \mathfrak{E}\right)$

It follows, in particular, that $h(T, Q \mid \mathfrak{E})=\lim _{i \rightarrow \infty} \frac{1}{\# F_{i}} H\left(\bigvee_{g \in F_{i}} T_{g}^{-1} Q \mid \mathfrak{E}\right)$ for each Følner sequence $\left(F_{i}\right)_{i \geq 1}$ in $G$.

Throughout this paper we use another-independent of $[\mathrm{Ki}, \mathrm{Ol}, \mathrm{OW}, \mathrm{RW}]$ definition for the E-relative entropy. In fact, we need Theorem 1.1 only in the proof of Theorem 3.3 just to show that the two definitions are equivalent. However, for completeness we demonstrate Theorem 1.1 in Appendix. Our proof is a slight 
modification of the argument from $[\mathrm{Ol}]$ and does not depend on the machinery from [OW2].

The E-relative entropy of $T$ is

$$
h(T \mid \mathfrak{E})=\sup \left\{h(T, Q \mid \mathfrak{E}) \mid Q \subset \mathfrak{B}_{Y}\right\}
$$

and the $\mathfrak{E}$-relative Pinsker algebra of $T$ is

$$
\Pi(T \mid \mathfrak{E})=\vee\left\{Q \subset \mathfrak{B}_{Y} \mid h(T, Q \mid \mathfrak{E})=0\right\} .
$$

If $\Pi(T \mid \mathfrak{E})=\mathfrak{E}$ then $T$ is called $\mathfrak{E}$-relatively $C P E$. We shall write $h(T)$ and $\Pi(T)$ instead of $h\left(T \mid \mathfrak{N}_{Y}\right)$ and $\Pi\left(T \mid \mathfrak{N}_{Y}\right)$ respectively.

Class-bijective factors (see also $[\mathrm{Da} 3, \S 1]$ ). We say that a $T$-factor $\mathfrak{E}$ is classbijective if for a measurable map $f: Y \rightarrow \mathbb{R}$ with $\mathfrak{E}=f^{-1}\left(\mathfrak{B}_{\mathbb{R}}\right)$, we have that $f$ is one-to-one on the $T$-orbits. Clearly if the factor-action $T \uparrow \mathfrak{E}$ is free then $\mathfrak{E}$ is class-bijective.

Given a cocycle $\beta$ of the $T$-orbit equivalence relation with values in $\operatorname{Aut}(Z, \kappa)$, denote by $T^{\beta}=\left\{\left(T_{g}\right)_{\beta}\right\}_{g \in G}$ the $\beta$-skew product extension of $T$. Then $\mathfrak{B}_{Y} \otimes \mathfrak{N}_{Z}$ is a class-bijective factor of $T^{\beta}$. Conversely, if $\mathfrak{E}$ is a class-bijective factor of an ergodic action $T$ then $T$ is isomorphic to a skew product extension of $T \nmid \mathfrak{E}$.

\section{Entropy And Pinsker Algebra For a COCYCle of A DISCRETE MEASURE PRESERVING EQUIVALENCE RELATION}

Given $\epsilon>0$ and two type I subrelations $\mathcal{T}$ and $\mathcal{S}$ of $\mathcal{R}$, we write $\mathcal{T} \subset_{\epsilon} \mathcal{S}$ if there is a subset $A \subset X, \mu(A)>1-\epsilon$, such that

$$
\#\left\{x^{\prime} \in \mathcal{S}(x) \mid \mathcal{T}\left(x^{\prime}\right) \subset \mathcal{S}(x)\right\}>(1-\epsilon) \# \mathcal{S}(x) \quad \text { for } x \in A .
$$

Replacing, if necessary, $A$ by $\bigcup_{x \in A} \mathcal{S}(x)$ we may (and will) assume that $A$ is $\mathcal{S}$ invariant. Let $A_{0}:=\{x \in A \mid \mathcal{T}(x) \subset \mathcal{S}(x)\}$. The following lemma is obvious.

Lemma 2.1. The subset $A_{0}$ is $\mathcal{T}$-invariant, $\mu\left(A_{0}\right)>1-2 \epsilon$ and $\#\left(\mathcal{S}(x) \cap A_{0}\right)>$ $(1-\epsilon) \# \mathcal{S}(x)$ for each $x \in A_{0}$.

Lemma 2.2. Let $\mathcal{R}$ be hyperfinite and $\left(\mathcal{R}_{n}\right)_{n \geq 1}$ a filtration of $\mathcal{R}$. Given $\epsilon>0$ and a countable subset $\Gamma \subset[\mathcal{R}]$ with $\#(\Gamma x)<\infty$ a.e., then for each sufficiently large $n$ there is an $\mathcal{R}_{n}$-invariant subset $A_{n}, \mu\left(A_{n}\right)>1-\epsilon$, with

$$
\#\left\{x^{\prime} \in \mathcal{R}_{n}(x) \mid \Gamma x^{\prime} \subset \mathcal{R}_{n}(x)\right\}>(1-\epsilon) \# \mathcal{R}_{n}(x) \quad \text { at every } x \in A_{n} .
$$

Proof. Given $f \in L^{1}(X, \mu)$ and a subrelation $\mathcal{S} \subset \mathcal{R}$, we denote by $E(f \mid \mathcal{S})$ the conditional expectation of $f$ with respect to the $\sigma$-algebra of $\mathcal{S}$-invariant subsets.

We first find $M>0$ and a subset $B, \mu(B)>1-\epsilon^{2}$, such that $\Gamma x \subset \mathcal{R}_{M}(x)$ for all $x \in B$. Clearly, $E\left(1_{B} \mid \mathcal{R}_{n}\right) \rightarrow E\left(1_{B} \mid \mathcal{R}\right)$ as $n \rightarrow \infty$. Next, $E\left(1_{B} \mid \mathcal{R}\right) \geq 0$ and $\int_{X} E\left(1_{B} \mid \mathcal{R}\right) d \mu=\mu(B)>1-\epsilon^{2}$. Hence there is $N>M$ such that for each $n>N$ there exists $A_{n} \subset X, \mu\left(A_{n}\right)>1-\epsilon$, with

$$
E\left(1_{B} \mid \mathcal{R}_{n}\right)(x)=\frac{\#\left(\mathcal{R}_{n}(x) \cap B\right)}{\# \mathcal{R}_{n}(x)}>1-\epsilon \quad \text { at all } x \in A_{n} .
$$

Without loss of generality we may assume that $A_{n}$ is $\mathcal{R}_{n}$-invariant. For each $x \in A_{n}$ and $x^{\prime} \in \mathcal{R}_{n}(x) \cap B$, we have $\Gamma x^{\prime} \subset \mathcal{R}_{M}\left(x^{\prime}\right)=\mathcal{R}_{M}(x) \subset \mathcal{R}_{n}(x)$. 
Corollary 2.3. Given $\epsilon>0$ and a type I subrelation $\mathcal{S}$ of $\mathcal{R}$, then $\mathcal{S} \subset_{\epsilon} \mathcal{R}_{n}$ for all sufficiently large $n$.

Let $\left(Y, \mathfrak{B}_{Y}, \nu\right)$ be a standard probability space, $\mathfrak{E}$ a sub- $\sigma$-algebra of $\mathfrak{B}_{Y}, \alpha$ : $\mathcal{R} \rightarrow \operatorname{Aut}_{\mathfrak{E}}(Y, \nu)$ a cocycle and $P$ a finite partition of $(X \times Y, \mu \times \nu)$. We consider $P$ as a measurable field $\left(P_{x}\right)_{x \in X}$ of finite $Y$-partitions, where $P_{x}=P \cap(\{x\} \times Y)$.

Definition 2.4. For a type I subrelation $\mathcal{S}$ of $\mathcal{R}$, we set

$$
h(\mathcal{S}, \alpha, P \mid \mathfrak{E}):=\int_{X} \frac{1}{\# \mathcal{S}(x)} H\left(\bigvee_{x^{\prime} \in \mathcal{S}(x)} \alpha\left(x, x^{\prime}\right) P_{x^{\prime}} \mid \mathfrak{E}\right) d \mu(x),
$$

and define the $\mathfrak{E}$-relative entropy of $(\alpha, P)$ as

$$
h(\alpha, P \mid \mathfrak{E}):=\inf \{h(S, \alpha, P \mid \mathfrak{E}) \mid \mathcal{S} \text { is a type I subrelation of } \mathcal{R}\}
$$

and the $\mathfrak{E}$-relative entropy of $\alpha$ as

$$
h(\alpha \mid \mathfrak{E}):=\sup \{h(\alpha, P \mid \mathfrak{E}) \mid P \text { is a finite partition of } X \times Y\} .
$$

We write $h(\alpha, P)$ and $h(\alpha)$ instead of $h\left(\alpha, P \mid \mathfrak{N}_{Y}\right)$ and $h\left(\alpha \mid \mathfrak{N}_{Y}\right)$ respectively.

Definition 2.5. By the $\mathfrak{E}$-relative Pinsker algebra of $\alpha$ we mean

$$
\Pi(\alpha \mid \mathfrak{E}):=\vee\left\{P \subset \mathfrak{B}_{X} \otimes \mathfrak{B}_{Y} \mid h(\alpha, P \mid \mathfrak{E})=0\right\} .
$$

Of course $\Pi(\alpha \mid \mathfrak{E}) \supset \mathfrak{B}_{X} \otimes \mathfrak{E}$. If $\Pi(\alpha \mid \mathfrak{E})=\mathfrak{B}_{X} \otimes \mathfrak{E}$ then $\alpha$ is called $\mathfrak{E}$-relatively CPE.

We shall exploit constantly the following two properties of the integrand in (2-1): it is $\mathcal{S}$-invariant and less than $\log (\# P)$.

Proposition 2.6. If $\mathcal{T} \subset_{\epsilon} \mathcal{S}$ then $h(\mathcal{S}, \alpha, P \mid \mathfrak{E}) \leq h(\mathcal{T}, \alpha, P \mid \mathfrak{E})+3 \epsilon \log (\# P)$.

Proof. Let $A_{0}$ be the subset from Lemma 2.1. We define two maps $f, g: A_{0} \rightarrow \mathbb{R}$ by setting

$$
\begin{aligned}
& f(x):=\frac{1}{\#\left(\mathcal{S}(x) \cap A_{0}\right)} H\left(\bigvee_{x^{\prime} \in \mathcal{S}(x) \cap A_{0}} \alpha\left(x, x^{\prime}\right) P_{x^{\prime}} \mid \mathfrak{E}\right) \\
& g(x):=\frac{1}{\# \mathcal{T}(x)} H\left(\bigvee_{x^{\prime} \in \mathcal{T}(x)} \alpha\left(x, x^{\prime}\right) P_{x^{\prime}} \mid \mathfrak{E}\right) .
\end{aligned}
$$

Since $A_{0}$ is $\mathcal{T}$-invariant, for each $x \in A_{0}$ there are $x_{1}, \ldots, x_{k} \in X$ with $\mathcal{S}(x) \cap A_{0}=$ $\bigsqcup_{i=1}^{k} \mathcal{T}\left(x_{i}\right)$. The sign $\bigsqcup$ denotes the union of disjoint subsets. It follows that

$$
\begin{aligned}
f(x) & \leq \frac{1}{\#\left(S(x) \cap A_{0}\right)} \sum_{i=1}^{k} H\left(\alpha\left(x_{i}, x\right) \bigvee_{x^{\prime} \in \mathcal{T}\left(x_{i}\right)} \alpha\left(x, x^{\prime}\right) P_{x^{\prime}} \mid \mathfrak{E}\right) \\
& =\frac{1}{\#\left(S(x) \cap A_{0}\right)} \sum_{i=1}^{k} \# \mathcal{T}\left(x_{i}\right) \cdot g\left(x_{i}\right) \\
& =\frac{1}{\#\left(S(x) \cap A_{0}\right)} \sum_{i=1}^{k} \sum_{x^{\prime} \in \mathcal{T}\left(x_{i}\right)} g\left(x^{\prime}\right) \\
& =\frac{1}{\#\left(S(x) \cap A_{0}\right)} \sum_{z \in \mathcal{S}(x) \cap A_{0}} g(z) \\
& =E\left(g \mid \mathcal{S} \cap\left(A_{0} \times A_{0}\right)\right)(x) .
\end{aligned}
$$


Hence

$$
\begin{aligned}
h(\mathcal{S}, \alpha, P \mid \mathfrak{E}) & \leq \int_{A_{0}}\left(f(x)+\frac{1}{\# \mathcal{S}(x)} H\left(\bigvee_{x^{\prime} \in \mathcal{S}(x) \backslash A_{0}} \alpha\left(x, x^{\prime}\right) P_{x^{\prime}} \mid \mathfrak{E}\right)\right) d \mu(x)+\epsilon \log (\# P) \\
& \leq \int_{A_{0}} E\left(g \mid \mathcal{S} \cap\left(A_{0} \times A_{0}\right)\right) d \mu+2 \epsilon \log (\# P) \\
& =\int_{A_{0}} g d \mu+2 \epsilon \log (\# P) \\
& \leq h(\mathcal{T}, \alpha, P \mid \mathfrak{E})+3 \epsilon \log (\# P) .
\end{aligned}
$$

The simplest application of this is that $h(\mathcal{S}, \alpha, P \mid \mathfrak{E}) \leq H\left(P \mid \mathfrak{B}_{X} \otimes \mathfrak{E}\right)$.

Corollary 2.7. Let $\mathcal{R}$ be hyperfinite and $\left(\mathcal{R}_{n}\right)_{n \geq 1}$ a filtration of $\mathcal{R}$. Then the sequence $h\left(\mathcal{R}_{n}, \alpha, P \mid \mathfrak{E}\right)$ converges to $h(\alpha, P \mid \mathfrak{E})$ as $n \rightarrow \infty$.

Proof. We deduce from Proposition 2.6 that $h\left(\mathcal{R}_{n}, \alpha, P \mid \mathfrak{E}\right)$ decreases and hence converges to some $a \geq h(\alpha, P \mid \mathfrak{E})$. The opposite inequality follows from Corollary 2.3 and Proposition 2.6.

Proposition 2.8. Let $\theta$ be a transformation from $N[\mathcal{R}]$ and $\phi: X \rightarrow \operatorname{Aut}_{\mathfrak{E}}(Y, \nu)$ a Borel map. Define two finite partitions $P^{\prime}=\left(P_{x}^{\prime}\right)_{x \in X}$ and $\left(P_{x}^{\prime \prime}\right)_{x \in X}$ of $X \times Y$ by setting $P_{x}^{\prime}:=\phi(x)^{-1} P_{x}$ and $P_{x}^{\prime \prime}:=P_{\theta^{-1} x}$. The following properties are satisfied:

(i) For a cocycle $\beta: \mathcal{R} \rightarrow$ Aut $_{\mathfrak{E}}(Y, \nu)$ given by $\beta \approx_{\phi} \alpha$, we have $h(\beta, P \mid \mathfrak{E})=$ $h\left(\alpha, P^{\prime} \mid \mathfrak{E}\right)$.

(ii) $h(\alpha \circ \theta, P \mid \mathfrak{E})=h\left(\alpha, P^{\prime \prime} \mid \mathfrak{E}\right)$.

Proof. Let $\mathcal{S}$ be a type $I$ subrelation of $\mathcal{R}$. Then

$$
\begin{aligned}
h(\mathcal{S}, \beta, P \mid \mathfrak{E}) & =\int_{X} \frac{1}{\# \mathcal{S}(x)} H\left(\bigvee_{x^{\prime} \in \mathcal{S}(x)} \phi(x) \alpha\left(x, x^{\prime}\right) \phi\left(x^{\prime}\right)^{-1} P_{x^{\prime}}\right) d \mu(x) \\
& =\int_{X} \frac{1}{\# \mathcal{S}(x)} H\left(\bigvee_{x^{\prime} \in \mathcal{S}(x)} \alpha\left(x, x^{\prime}\right) P_{x^{\prime}}^{\prime}\right) d \mu(x) \\
& =h\left(\mathcal{S}, \alpha, P^{\prime} \mid \mathfrak{E}\right)
\end{aligned}
$$

and (i) follows.

To prove (ii), we let $\mathcal{T}:=(\theta \times \theta) \mathcal{S}$. It is clear that $\mathcal{T}$ is a type $I$ subrelation of $\mathcal{R}$ and $\mathcal{T}(x)=\theta \mathcal{S}\left(\theta^{-1} x\right)$ for all $x \in X$. Hence

$$
\begin{aligned}
h(\mathcal{S}, \alpha \circ \theta, P \mid \mathfrak{E}) & =\int_{X} \frac{1}{\# \mathcal{S}(x)} H\left(\bigvee_{x^{\prime} \in \mathcal{S}(x)} \alpha\left(\theta x, \theta x^{\prime}\right) P_{x^{\prime}}\right) d \mu(x) \\
& =\int_{X} \frac{1}{\# \mathcal{S}\left(\theta^{-1} z\right)} H\left(\bigvee_{\theta x^{\prime} \in \mathcal{T}(z)} \alpha\left(z, \theta x^{\prime}\right) P_{x^{\prime}}\right) d \mu(z) \\
& =\int_{X} \frac{1}{\# \mathcal{T}(z)} H\left(\bigvee_{z^{\prime} \in \mathcal{T}(z)} \alpha\left(z, z^{\prime}\right) P_{z^{\prime}}^{\prime \prime}\right) d \mu(z) \\
& =h\left(\mathcal{T}, \alpha, P^{\prime \prime} \mid \mathfrak{E}\right) .
\end{aligned}
$$

Since the map $\mathcal{S} \mapsto \mathcal{T}$ is a bijection on the set of type $I \mathcal{R}$-subrelations, (ii) follows. 
Corollary 2.9. Let two cocycles $\alpha, \beta: \mathcal{R} \rightarrow A u t_{\mathfrak{E}}(Y, \nu)$ are cohomologous or weakly equivalent. The following properties are satisfied:

(i) $h(\alpha \mid \mathfrak{E})=h(\beta \mid \mathfrak{E})$.

(ii) If $\alpha$ is $\mathfrak{E}$-relatively $C P E$ then $\beta$ so is.

Definition 2.10 ([DaG], [Da1]). A transformation $\theta \in N[\mathcal{R}]$ is called compatible with $\alpha$ if $\alpha \circ \theta$ is cohomologous to $\alpha$.

Every transformation $\gamma \in[\mathcal{R}]$ is compatible with $\alpha-\operatorname{set} \phi(x):=\alpha(\gamma x, x), x \in X$. We let

$$
\widetilde{D}(\mathcal{R}, \alpha):=\left\{\theta_{\phi} \mid \alpha \circ \theta \approx_{\phi} \alpha\right\},
$$

where $\theta_{\phi}$ is a $\mu \times \nu$-preserving transformation of $X \times Y$, defined by $\theta_{\phi}(x, y)=$ $(\theta x, \phi(x) y)$. It is a routine to verify that $\widetilde{D}(\mathcal{R}, \alpha)$ is a subgroup of $N[\mathcal{R}(\alpha)]$.

Corollary 2.11. The E-Eelative Pinsker algebra of $\alpha$ is $\widetilde{D}(\mathcal{R}, \alpha)$-invariant.

Proof. Let $\alpha \circ \theta \approx_{\phi} \alpha$. It is easy to verify that $\left(\theta_{\phi} P\right)_{x}=\phi\left(\theta^{-1} x\right) P_{\theta^{-1} x}$ for all $x \in X$. ¿From Proposition 2.8 we deduce that

$$
h\left(\alpha, \theta_{\phi} P \mid \mathfrak{E}\right)=h\left(\alpha \circ \theta,\left(\phi(x) P_{x}\right)_{x \in X} \mid \mathfrak{E}\right)=h(\alpha, P \mid \mathfrak{E}) .
$$

Hence $P \in \Pi(\alpha \mid \mathfrak{E})$ if and only if $\theta_{\phi} P \in \Pi(\alpha \mid \mathfrak{E})$.

The following statement is an orbital counterpart of [RW, Theorem 4.10]. Here we adapt their proof.

Theorem 2.12. If there exists an ergodic countable subgroup $\Gamma$ of $\alpha$-compatible transformations such that $\alpha \circ \gamma=\alpha$ for all $\gamma \in \Gamma$ then $\Pi(\alpha \mid \mathfrak{E})=\mathfrak{B}_{X} \otimes \mathfrak{F}$ for a sub- $\sigma$-algebra $\mathfrak{F} \supset \mathfrak{E}$ of $\mathfrak{B}_{Y}$.

Proof. Denote by $\mathfrak{F}_{x}$ the restriction of $\Pi(\alpha \mid \mathfrak{E})$ onto $\{x\} \times Y, x \in X$. It is well known that the space $\Sigma$ of sub- $\sigma$-algebras of $\mathfrak{B}_{Y}$ is Polish and the map $X \ni x \mapsto$ $\mathfrak{F}_{x} \in \Sigma$ is measurable. It follows from our assumption on $\alpha$ and Corollary 2.11 that $(\gamma \times$ id $) \mathfrak{F}=\mathfrak{F}$ and hence $\mathfrak{F}_{\gamma x}=\mathfrak{F}_{x} \mu$-a.e. for each $\gamma \in \Gamma$. By the ergodicity of $\Gamma$ there exists $\mathfrak{F} \in \Sigma$ with $\mathfrak{F}_{x}=\mathfrak{F}$ a.e. Since $\Pi(\alpha \mid \mathfrak{E}) \supset \mathfrak{B}_{X} \otimes \mathfrak{N}_{Y}$, we deduce that $\Pi(\alpha \mid \mathfrak{E})=\mathfrak{B}_{X} \otimes \mathfrak{F}$. Clearly, $\mathfrak{F} \supset \mathfrak{E}$.

Theorem 2.13. If $\alpha$ is recurrent then $h(\alpha \mid \mathfrak{E})=0$, i.e. $\Pi(\alpha \mid \mathfrak{E})=\mathfrak{B}_{X} \otimes \mathfrak{B}_{Y}$.

To prove this theorem we need two auxiliary lemmas.

Lemma 2.14 [GS, Proposition 1.1, Da2, Lemma 1.5]. Let $A$ be a Polish group, $\mathcal{S}$ a hyperfinite discrete equivalence relation on $(X, \mathfrak{B}, \mu)$ and $\alpha, \beta: \mathcal{S} \rightarrow A$ two cocycles. For a filtration $\left(\mathcal{S}_{n}\right)_{n \geq 1}$ of $\mathcal{S}$, consider two sequences of Borel maps $a_{n}, b_{n}: X \rightarrow A$ such that $\alpha(x, y)=a_{n}(x) a_{n}(y)^{-1}$ and $\beta(x, y)=b_{n}(x) b_{n}(y)^{-1}$ for all $(x, y) \in \mathcal{S}_{n}$. If $a_{n}(x) b_{n}(x)^{-1}$ converges a.e. to a map $\phi: X \rightarrow A$ as $n \rightarrow \infty$ then $\alpha \approx_{\phi} \beta$.

Lemma 2.15. Let $A$ be a Polish group and $\alpha: \mathcal{R} \rightarrow A$ a recurrent cocycle. Then there exist a hyperfinite conservative subrelation $\mathcal{S} \subset \mathcal{R}$ and a $\alpha$-cohomologous cocycle $\beta: \mathcal{R} \rightarrow A$ such that $\beta(\mathcal{S})=1_{A}$.

Proof (cf. [Da2, Proposition 1.8], [GoS, Proposition 1.2]). Let $\left(W_{n}\right)_{n}$ be a fundamental system of symmetric neighborhoods of $1_{A}$ such that $W_{n+1} W_{n+1} W_{n+1} \subset W_{n}$ 
for all $n$. Since $\alpha$ is recurrent, we apply a standard exhaustion argument to construct a sequence of Borel sets $\left(F_{n}\right)_{n \geq 0}$ such that $F_{0} \supset F_{1} \supset \cdots$ and $\mu\left(F_{n}\right)=2^{-n}$ and a sequence of Borel isomorphisms $t_{n}: F_{n-1} \backslash F_{n} \rightarrow F_{n}$ such that $\left(x, t_{n} x\right) \in \mathcal{R}$ and $\alpha\left(x, t_{n} x\right) \in W_{n}$. We define inductively Borel maps $T_{n}: X \rightarrow F_{n}$ by setting

$$
T_{n} x:= \begin{cases}x & \text { for } x \in F_{n} \\ t_{n} T_{n-1} \cdots T_{1} & \text { otherwise }\end{cases}
$$

Then we let $\mathcal{S}_{n}:=\left\{(x, y) \mid T_{n} x=T_{n} y\right\}$. Clearly, $\mathcal{S}_{1} \subset \mathcal{S}_{2} \subset \cdots \subset \mathcal{R}, \# \mathcal{S}_{n}(x)=2^{n}$ for a.e. $x \in X$ and $F_{n}$ is an $\mathcal{S}_{n}$-fundamental domain. Hence the equivalence relation $\mathcal{S}:=\bigcup_{n} \mathcal{S}_{n}$ is hyperfinite and countable (i.e. conservative). To complete the proof it is sufficient to show that $\alpha$ restricted to $\mathcal{S}$ is a coboundary. Notice that $\alpha(x, y)=\alpha\left(x, T_{n} x\right) \alpha\left(y, T_{n} y\right)^{-1}$ for all $(x, y) \in \mathcal{S}_{n}$ and

$$
\begin{aligned}
\alpha\left(x, T_{n+k} x\right) & =\alpha\left(x, T_{n+k-1} x\right) \alpha\left(T_{n+k-1} x, t_{n+k} T_{n+k-1} x\right) \\
& \in \alpha\left(x, T_{n+k-1} x\right) W_{n+k} \\
& \subset \cdots \subset \alpha\left(x, T_{n} x\right) W_{n+1} W_{n+2} \cdots W_{n+k} \\
& \subset \alpha\left(x, T_{n} x\right) W_{n} .
\end{aligned}
$$

It remains to apply Lemma 2.14 with $a_{n}(x):=\alpha\left(x, T_{n} x\right)$ and $b_{n}(x):=1_{A}$.

Proof of Theorem 2.13. By Lemma 2.15, there exist a hyperfinite countable subrelation $\mathcal{S} \subset \mathcal{R}$ and an $\alpha$-compatible cocycle $\beta: \mathcal{R} \rightarrow \operatorname{Aut}_{\mathfrak{E}}(Y, \nu)$ such that $\beta(\mathcal{S})=\operatorname{Id}_{Y}$. If follows from Corollary 2.9 that $h(\alpha \mid \mathfrak{E})=h(\beta \mid \mathfrak{E})$. Moreover, for each finite partition $P$ of $X \times Y$, we have

$$
h(\beta, P \mid \mathfrak{E}) \leq \inf \{h(\mathcal{T}, \beta, P \mid \mathfrak{E}) \mid \mathcal{T} \text { is a type I subrelation of } S\}
$$

and

$$
h(\mathcal{T}, \beta, P \mid \mathfrak{E})=\int_{X} \frac{1}{\# \mathcal{T}(x)} H\left(P_{x} \mid \mathfrak{E}\right) d \mu(x) \leq \frac{\log \# P}{\min _{x \in X} \# \mathcal{T}(x)} .
$$

Since $\mathcal{T}$ is hyperfinite and countable, vrai $\min _{x \in X} \# \mathcal{S}_{n}(x) \rightarrow \infty$ for any filtration $\left(\mathcal{S}_{n}\right)_{n}$ of $\mathcal{S}$. Hence $h(\beta, P \mid \mathfrak{E})=0$ which implies $h(\beta \mid \mathfrak{E})=0$.

Let $\mathcal{R}$ be ergodic. Then for each subrelation $\mathcal{S}$ in $\mathcal{R}$, there exists $n \in \mathbb{N} \cup\{\infty\}$ such that a.e. $\mathcal{R}$-class consists of $n$ different $\mathcal{S}$-classes [FSZ]. This number is called the index of $\mathcal{S}$ in $\mathcal{R}$. We denote it by $\operatorname{ind}(\mathcal{R}: \mathcal{S})$.

Theorem 2.16. Let $\mathcal{R}$ be hyperfinite and $\mathcal{S}$ an ergodic $\mathcal{R}$-subrelation of finite index. Then $h((\alpha\lceil\mathcal{S}) \mid \mathfrak{E})=\operatorname{ind}(\mathcal{R}: \mathcal{S}) \cdot h(\alpha \mid \mathfrak{E})$, where $\alpha\lceil\mathcal{S}$ stands for the restriction of $\alpha$ to $\mathcal{S}$.

Proof. It follows from [FSZ] that there exist an ergodic subrelation $\mathcal{T}$ in $\mathcal{S}$ and two nested finite subgroups $H \subset G$ in $N[\mathcal{T}]$ such that:

(o) $G \cap[\mathcal{T}]=\{\operatorname{Id}\}$,

(o) $H$ contains no nontrivial normal subgroups of $G$,

(o) $\mathcal{R}$ is generated by $\mathcal{T}$ and $G$,

(o) $\mathcal{S}$ is generated by $\mathcal{T}$ and $H$,

(o) $\operatorname{ind}(\mathcal{R}: \mathcal{S})=\# G / \# H$. 
Via the standard outer conjugacy trick one can find a filtration $\left(\mathcal{T}_{n}\right)_{n \geq 1}$ of $\mathcal{T}$ such that $G \subset \bigcap_{n} N\left[\mathcal{I}_{n}\right]$. Denote by $\mathcal{R}_{n}$ (resp. $\mathcal{S}_{n}$ ) the equivalence relation generated by $\mathcal{T}_{n}$ and $G$ (resp. $H$ ). Then $\left(\mathcal{R}_{n}\right)_{n}$ is a filtration of $\mathcal{R}$ and $\left(\mathcal{S}_{n}\right)_{n}$ is a filtration of $\mathcal{S}$. For a finite partition $P$ of $X \times Y$, we let $P^{G}:=\bigvee_{\gamma \in G} \gamma_{\alpha}^{-1} P$ and $P^{H}:=$ $\bigvee_{\gamma \in H} \gamma_{\alpha}^{-1} P$. Recall that $\gamma_{\alpha}$ is the $\alpha$-skew product extension of $\gamma$ (see $\S 1$ ). Since $\bigsqcup_{\gamma \in G} \gamma \mathcal{T}_{n}(x)=\bigsqcup_{\gamma \in G} \mathcal{T}_{n}(\gamma x)=\mathcal{R}_{n}(x)$, we have $\# \mathcal{R}_{n}(x)=\# G \# \mathcal{T}_{n}(x)$ and

$$
\bigvee_{x^{\prime} \in \mathcal{T}_{n}(x)} \alpha\left(x, x^{\prime}\right) P_{x^{\prime}}^{G}=\bigvee_{x^{\prime} \in \mathcal{T}_{n}(x)} \alpha\left(x, x^{\prime}\right) \bigvee_{\gamma \in G} \alpha\left(x^{\prime}, \gamma x^{\prime}\right) P_{\gamma x^{\prime}}=\bigvee_{z \in \mathcal{R}_{n}(x)} \alpha(x, z) P_{z}
$$

for a.e. $x \in X$. Hence

$$
\begin{aligned}
h\left(\mathcal{T}_{n}, \alpha, P^{G} \mid \mathfrak{E}\right) & =\int_{X} \frac{1}{\# \mathcal{T}_{n}(x)} H\left(\bigvee_{x^{\prime} \in \mathcal{T}_{n}(x)} \alpha\left(x, x^{\prime}\right) P_{x^{\prime}}^{G}\right) d \mu(x) \\
& =\# G \int_{X} \frac{1}{\# G \# \mathcal{T}_{n}(x)} H\left(\bigvee_{z \in \mathcal{R}_{n}(x)} \alpha(x, z) P_{z}\right) d \mu(x) \\
& =\# G \cdot h\left(\mathcal{R}_{n}, \alpha, P \mid \mathfrak{E}\right) .
\end{aligned}
$$

It follows that $h\left(\alpha \uparrow \mathcal{T}, P^{G} \mid \mathfrak{E}\right)=\# G \cdot h(\alpha, P \mid \mathfrak{E})$. From this we deduce that

$$
\begin{aligned}
h((\alpha \uparrow \mathcal{T}) \mid \mathfrak{E}) & =\sup \{h(\alpha \uparrow \mathcal{T}, P \mid \mathfrak{E}) \mid P \text { is a finite partition of } X \times Y\} \\
& =\sup \left\{h\left(\alpha \uparrow \mathcal{T}, P^{G} \mid \mathfrak{E}\right) \mid P \text { is a finite partition of } X \times Y\right\} \\
& =\# G \cdot h(\alpha \mid \mathfrak{E}) .
\end{aligned}
$$

In a similar way, $h((\alpha \uparrow \mathcal{T}) \mid \mathfrak{E})=\# H \cdot h((\alpha \uparrow \mathcal{S}) \mid \mathfrak{E})$. Hence $h((\alpha\lceil\mathcal{S}) \mid \mathfrak{E})=$ $\frac{\# G}{\# H} h(\alpha \mid \mathfrak{E})$, as desired.

Definition 2.17. The fiber entropy of $\alpha$ is

$$
h_{\mathrm{fib}}(\alpha \mid \mathfrak{E}):=\sup \left\{h\left(\alpha, \mathfrak{N}_{X} \otimes Q \mid \mathfrak{E}\right) \mid Q \text { is a finite } Y \text {-partition }\right\} .
$$

We write $h_{\text {fib }}(\alpha)$ instead of $h_{\mathrm{fib}}\left(\alpha \mid \mathfrak{N}_{Y}\right)$.

Lemma 2.18. $h_{\text {fib }}(\alpha \mid \mathfrak{E})=h(\alpha \mid \mathfrak{E})$.

Proof. It is easy to verify that

$h(\alpha \mid \mathfrak{E})=\sup \{h(\alpha, P \otimes Q \mid \mathfrak{E}) \mid P, Q$ are finite partitions of $X$ and $Y$ respectively $\}$

For a type I subrelation $\mathcal{S}$ of $\mathcal{R}$, we have

$$
h\left(\mathcal{S}, \alpha,\left(P \otimes \mathfrak{N}_{Y}\right) \vee\left(\mathfrak{N}_{X} \otimes Q\right) \mid \mathfrak{E}\right)=h\left(\mathcal{S}, \alpha, \mathfrak{N}_{X} \otimes Q \mid \mathfrak{E}\right)
$$

and hence $h(\alpha, P \otimes Q \mid \mathfrak{E})=h\left(\alpha, \mathfrak{N}_{X} \otimes Q \mid \mathfrak{E}\right)$. 


\section{Relatively CPE-ACtions are Uniformly Relatively mixing}

In this section our main goal is to prove Theorem 0.1 without the basic machinery developed in $[\mathrm{OW}]$. For this we first provide a new definition for the relative entropy of a process which is well suited for the techniques from $\S 2$. We shall show that this definition is equivalent to the standard one given in $\S 1$. An important Theorem 3.6 establishes a connection of the orbital concept $h(\alpha, P \mid \mathfrak{E})$ with the classical conditional entropy of a transformation (i.e. $\mathbb{Z}$-action).

Everywhere below $G$ is a countable amenable group and $G \times X \ni(g, x) \mapsto g x \in X$ a free $G$-action which generates $\mathcal{R}$. It follows that $\mathcal{R}$ is hyperfinite and conservative. Given a type I subrelation $\mathcal{S} \subset \mathcal{R}$, let a subset $B \subset X$ be an $\mathcal{S}$-fundamental domain. Then there is a measurable map $B \ni x \mapsto G_{x} \in \operatorname{Fin}(G)$ such that $G_{x} x=\mathcal{S}(x)$ and hence $X=\bigsqcup_{x \in B} G_{x} x$. Since $\operatorname{Fin}(\mathrm{G})$ is countable, we obtain that $X=\bigsqcup_{i} \bigsqcup_{g \in G_{i}} g B_{i}$ for a countable family $G_{i} \in \operatorname{Fin}(G)$ and a decomposition $B=\bigsqcup_{i} B_{i}$ with $G_{i} x=\mathcal{S}(x)$ at all $x \in B_{i}$. We shall write $\mathcal{S} \sim\left(B_{i}, G_{i}\right)$. Of course

$$
\begin{aligned}
h(\mathcal{S}, \alpha, P \mid \mathfrak{E}) & =\sum_{i} \sum_{g \in G_{i}} \int_{g B_{i}} \frac{1}{\# G_{i}} H\left(\bigvee_{x^{\prime} \in \mathcal{S}(x)} \alpha\left(x, x^{\prime}\right) P_{x^{\prime}} \mid \mathfrak{E}\right) d \mu(x) \\
& =\sum_{i} \int_{B_{i}} H\left(\bigvee_{g \in G_{i}} \alpha(x, g x) P_{g x} \mid \mathfrak{E}\right) d \mu(x)
\end{aligned}
$$

Definition 3.1. Let $T$ be an action of $G$ on $\left(Y, \mathfrak{B}_{Y}, \nu\right)$, $\mathfrak{E}$ a factor of $T$ and $Q$ a finite partition of $Y$. We define the virtual $\mathfrak{E}$-relative entropy of the process $(T, Q)$ as $\widehat{h}(T, Q \mid \mathfrak{E}):=h\left(\beta_{T}, \mathfrak{N}_{X} \otimes Q \mid \mathfrak{E}\right)$, where $\beta_{T}: \mathcal{R} \rightarrow \operatorname{Aut}_{\mathfrak{E}}(Y, \nu)$ is a cocycle given by $\beta_{T}(g x, x)=T_{g}$.

Since there are plenty of free $G$-actions generating $\mathcal{R}$, the cocycle $\beta_{T}$ is not determined uniquely by $(T, Q)$. Hence we need to verify that $\widehat{h}(T, Q \mid \mathfrak{E})$ is well defined.

Proposition 3.2. Let $\left\{U_{g}\right\}_{g \in G}$ and $\left\{U_{g}^{\prime}\right\}_{g \in G}$ be two free $G$-actions on $\left(X, \mathfrak{B}_{X}, \mu\right)$ such that $\left\{U_{g} x \mid g \in G\right\}=\left\{U_{g}^{\prime} x \mid g \in G\right\}=\mathcal{R}(x)$ for a.e. $x \in X$. Define two cocycles $\beta, \beta^{\prime}: \mathcal{R} \rightarrow \operatorname{Aut}_{\mathfrak{E}}(Y, \nu)$ by setting

$$
\beta\left(U_{g} x, x\right)=\beta^{\prime}\left(U_{g}^{\prime} x, x\right)=T_{g}, \quad g \in G, x \in X .
$$

Then $h\left(\beta, \mathfrak{N}_{X} \otimes Q \mid \mathfrak{E}\right)=h\left(\beta^{\prime}, \mathfrak{N}_{X} \otimes Q \mid \mathfrak{E}\right)$.

Proof. Denote by $\mathcal{S}$ the equivalence relation on $(X \times X, \mu \times \mu)$ generated by the diagonal $G$-action $\left\{U_{g} \times U_{g}^{\prime}\right\}_{g \in G}$. Clearly, $\mathcal{S}$ is measure preserving and hyperfinite. Let $\alpha_{U}, \alpha_{U^{\prime}}: \mathcal{R} \rightarrow \operatorname{Aut}(X, \mu)$ and $\beta_{T}: \mathcal{S} \rightarrow \operatorname{Aut}_{\mathfrak{E}}(Y, \nu)$ be cocycles defined by

$$
\begin{aligned}
\alpha_{U}\left(U_{g}^{\prime} x, x\right) & :=U_{g}, \\
\alpha_{U^{\prime}}\left(U_{g} x, x\right) & :=U_{g}^{\prime}, \\
\beta_{T}\left(U_{g} x, U_{g}^{\prime} x^{\prime}, x, x^{\prime}\right) & :=T_{g},
\end{aligned}
$$

for all $g \in G, x, x^{\prime} \in X$. It is easy to see that $\mathcal{S}=\mathcal{R}\left(\alpha_{U^{\prime}}\right)=\sigma \mathcal{R}\left(\alpha_{U}\right) \sigma$, where $\sigma: X \times X \rightarrow X \times X$ is the flip, i.e. $\sigma\left(x, x^{\prime}\right)=\left(x^{\prime}, x\right)$ (we refer the reader to $\S 1$ for the definition of the skew product extensions $\mathcal{R}\left(\alpha_{U^{\prime}}\right)$ and $\left.\mathcal{R}\left(\alpha_{U}\right)\right)$. Hence 
if $\left(\mathcal{R}_{n}\right)_{n \geq 1}$ is a filtration of $\mathcal{R}$ then $\left(\mathcal{R}_{n}\left(\alpha_{U^{\prime}}\right)\right)_{n \geq 1}$ and $\left(\sigma \mathcal{R}_{n}\left(\alpha_{U}\right) \sigma\right)_{n \geq 1}$ are two filtrations of $\mathcal{S}$. It is easily verified that

$$
\begin{aligned}
h\left(\mathcal{R}_{n}\left(\alpha_{U^{\prime}}\right), \beta_{T}, \mathfrak{N}_{X \times X} \otimes Q \mid \mathfrak{E}\right) & =h\left(\mathcal{R}_{n}, \beta, \mathfrak{N}_{X} \otimes Q \mid \mathfrak{E}\right), \\
h\left(\sigma \mathcal{R}_{n}\left(\alpha_{U}\right) \sigma, \beta_{T}, \mathfrak{N}_{X \times X} \otimes Q \mid \mathfrak{E}\right) & =h\left(\mathcal{R}_{n}, \beta^{\prime}, \mathfrak{N}_{X} \otimes Q \mid \mathfrak{E}\right) .
\end{aligned}
$$

Passing to the limit we obtain

$$
\begin{aligned}
& h\left(\beta_{T}, \mathfrak{N}_{X \times X} \otimes Q \mid \mathfrak{E}\right)=h\left(\beta, \mathfrak{N}_{X} \otimes Q \mid \mathfrak{E}\right), \\
& h\left(\beta_{T}, \mathfrak{N}_{X \times X} \otimes Q \mid \mathfrak{E}\right)=h\left(\beta^{\prime}, \mathfrak{N}_{X} \otimes Q \mid \mathfrak{E}\right) .
\end{aligned}
$$

\section{Theorem 3.3.}

(i) $h(T, Q \mid \mathfrak{E})=\widehat{h}(T, Q \mid \mathfrak{E})$,

(ii) $h(T \mid \mathfrak{E})=h\left(\beta_{T} \mid \mathfrak{E}\right)$.

Proof. (i) By Theorem 1.1, for each $\epsilon>0$, there exists $\epsilon^{\prime}, 0<\epsilon^{\prime}<\epsilon$, and a finite subset $K \subset G$ with

$$
\left|\frac{1}{\# F} H\left(\bigvee_{g \in F} T_{g}^{-1} Q \mid \mathfrak{E}\right)-h(T, Q \mid \mathfrak{E})\right|<\epsilon
$$

for every $\left[K, \epsilon^{\prime}\right]$-invariant subset $F \subset G$. Let $\left(\mathcal{R}_{n}\right)_{n \geq 1}$ be a filtration of $\mathcal{R}$ and $\mathcal{R}_{n} \sim\left(B_{i}^{(n)}, G_{i}^{(n)}\right)$. By Lemma 2.2, for each sufficiently large $n$ there is a subset $A_{n} \subset X, \mu\left(A_{n}\right)>1-\epsilon^{\prime}$, with

$$
\#\left\{x^{\prime} \in \mathcal{R}_{n}(x) \mid K x^{\prime} \subset \mathcal{R}_{n}(x)\right\}>\left(1-\epsilon^{\prime}\right) \# \mathcal{R}_{n}(x) \quad \text { at all } x \in A_{n} .
$$

Since $A_{n}$ is $\mathcal{R}_{n}$-invariant (by Lemma 2.2), $A_{n}=\bigsqcup_{i \in J} G_{i}^{(n)} C_{i}^{(n)}$ for some subset $J \subset \mathbb{N}$ and a family of measurable subsets $C_{i}^{(n)} \subset B_{i}^{(n)}$ with $\mu\left(C_{i}^{(n)}\right)>0$. We deduce from (3-2) that $G_{i}^{(n)}$ is $\left[K, \epsilon^{\prime}\right]$-invariant for each $i \in J$. Hence

$$
\begin{aligned}
h\left(\mathcal{R}_{n}, \beta_{T}, \mathfrak{N}_{X} \otimes Q \mid \mathfrak{E}\right) & =\int_{A_{n}}+\int_{X \backslash A_{n}} \\
& =\sum_{j \in J} \int_{C_{j}^{(n)}} H\left(\bigvee_{g \in G_{j}^{(n)}} T_{g}^{-1} Q \mid \mathfrak{E}\right) d \mu(x) \pm \epsilon^{\prime} \log (\# Q) \\
& =(h(T, Q \mid \mathfrak{E}) \pm \epsilon) \mu\left(A_{n}\right) \pm \epsilon \log (\# Q) .
\end{aligned}
$$

Passing to the limit we obtain $\widehat{h}(T, Q \mid \mathfrak{E}):=h\left(\beta_{T}, \mathfrak{N}_{X} \otimes Q \mid \mathfrak{E}\right)=h(T, Q \mid \mathfrak{E})$.

(ii) follows from (i) and Lemma 2.18.

Remark that Theorem 3.3(i) provides once more proof of the fact that the virtual $\mathfrak{E}$-relative entropy is well defined.

Corollary 3.4. If the centralizer $C(G)$ of the $G$-action in $\operatorname{Aut}(X, \mu)$ is ergodic then $\Pi\left(\beta_{T} \mid \mathfrak{E}\right)=\mathfrak{B}_{X} \otimes \Pi(T \mid \mathfrak{E})$. Hence $\beta_{T}$ is $\mathfrak{E}$-relatively $C P E$ if and only if $T$ so is. (Recall that $G$ is embedded into $[\mathcal{R}] \subset \operatorname{Aut}(X, \mu)$.)

Proof. Since $C(G) \subset N[\mathcal{R}]$ and $\beta_{T}(\gamma x, \gamma g x)=\beta_{T}(x, g x)$ a.e. for each $\gamma \in C(G)$ and $g \in G$ at a.e. $x \in X$, it follows from Theorem 2.12 that $\Pi\left(\beta_{T} \mid \mathfrak{E}\right)=\mathfrak{B}_{X} \otimes \mathfrak{F}$ for a sub- $\sigma$-algebra $\mathfrak{F} \subset \mathfrak{B}_{Y}$. Hence a finite $Y$-partition $Q$ is $\mathfrak{F}$-measurable if and only if $\mathfrak{N}_{X} \otimes Q$ is $\Pi\left(\beta_{T} \mid \mathfrak{E}\right)$-measurable, i.e. when $h\left(\beta_{T}, \mathfrak{N}_{X} \otimes Q \mid \mathfrak{E}\right)=0$. By Theorem 3.3 the last is equivalent to $h(T, Q \mid \mathfrak{E})=0$, i.e. $Q$ is $\Pi(T \mid \mathfrak{E})$-measurable. 
Corollary 3.5 (Entropy of a subgroup action). Let $H$ be a subgroup of finite index in $G$. Then $h(T(H) \mid \mathfrak{E})=\#(G / H) h(T \mid \mathfrak{E})$, where $T(H)=\left\{T_{g}\right\}_{g \in H}$.

Proof. Let $\mathcal{R}$ be generated by a Bernoullian $G$-action. Denote by $\mathcal{S}$ the $H$-orbit subrelation. Then $\mathcal{R}$ is hyperfinite, $\mathcal{S}$ is ergodic and $\operatorname{ind}(\mathcal{R}: \mathcal{S})=\#(G / H)$. Let $\beta_{T}: \mathcal{R} \rightarrow \operatorname{Aut}_{\mathfrak{E}}(Y, \nu)$ be the cocycle determined in Definition 3.1. By Theorem 2.16,

$$
h\left(\left(\beta_{T}\lceil\mathcal{S}) \mid \mathfrak{E}\right)=\operatorname{ind}(\mathcal{R}: \mathcal{S}) \cdot h\left(\beta_{T} \mid \mathfrak{E}\right)\right.
$$

and we deduce from Theorem 3.3 that $h(T(H) \mid \mathfrak{E})=\#(G / H) \cdot h(T \mid \mathfrak{E})$.

Theorem 3.6. Let $\gamma$ be a transformation generating $\mathcal{R}$ and $\gamma_{\alpha}$ stand for the $\alpha$-skew product extension of $\gamma$. Then $h(\alpha, P \mid \mathfrak{E})=h\left(\gamma_{\alpha}, P \mid \mathfrak{B}_{X} \otimes \mathfrak{E}\right)$.

Proof. Let $\delta$ be an ergodic transformation on a standard probability space $(Z, \kappa)$ with the pure point 2 -adic rational spectrum. Denote by $\mathcal{S}$ the $(\delta \times \gamma)$-orbit equivalence relation on $Z \times X$. Let $\sigma: Z \times X \rightarrow X \times Z$ stand for the flip. We have that $\mathcal{S}=\sigma^{-1} \mathcal{R}(\beta) \sigma$ for the cocycle $\beta: \mathcal{R} \rightarrow \operatorname{Aut}(Z, \kappa)$ given by $\beta\left(\gamma^{n} x, x\right)=\delta^{n}, n \in \mathbb{Z}$. Since $\mathcal{R}$ is conservative, $\gamma$ is aperiodic and hence $\beta$ is well defined.

Now we define a cocycle $1 \otimes \alpha: \mathcal{S} \rightarrow \operatorname{Aut}_{\mathfrak{E}}(Y, \nu)$ by setting

$$
1 \otimes \alpha\left((z, x),\left(z^{\prime}, x^{\prime}\right)\right)=\alpha\left(x, x^{\prime}\right)
$$

If $\left(\mathcal{R}_{n}\right)_{n \geq 1}$ is a filtration of $\mathcal{R}$ then $\left(\sigma^{-1} \mathcal{R}_{n}(\beta) \sigma\right)_{n \geq 1}$ is a filtration of $\mathcal{S}$. Hence

$$
\begin{aligned}
h\left(1 \otimes \alpha, \mathfrak{N}_{Z} \otimes P \mid \mathfrak{E}\right) & =\lim _{n \rightarrow \infty} h\left(\sigma^{-1} \mathcal{R}_{n}(\beta) \sigma, 1 \otimes \alpha, \mathfrak{N}_{Z} \otimes P \mid \mathfrak{E}\right) \\
& =\lim _{n \rightarrow \infty} h\left(\mathcal{R}_{n}, \alpha, P \mid \mathfrak{E}\right)=h(\alpha, P \mid \mathfrak{E}) .
\end{aligned}
$$

On the other hand, it follows from the assumptions on $\delta$ that there is a sequence $A_{1} \subset A_{2} \subset \ldots$ of measurable subsets of $Z$ such that $Z=\bigsqcup_{i=0}^{2^{n}-1} \delta^{i} A_{n}$ for each $n \geq 1$. Hence $Z \times X=\bigsqcup_{i=0}^{2^{n}-1}(\delta \times \gamma)^{i}\left(A_{n} \times X\right)$. Notice that $\kappa\left(A_{n}\right)=2^{-n}$. Denote by $\mathcal{S}_{n}$ the type I subrelation of $\mathcal{S}$ such that

$$
\mathcal{S}_{n} \sim\left(A_{n} \times X,\left\{(\delta \times \gamma)^{i} \mid i=0, \ldots 2^{n}-1\right\}\right) .
$$

Clearly $\mathcal{S}_{1} \subset \mathcal{S}_{2} \subset \ldots$ and $\bigcup_{n} \mathcal{S}_{n}=\mathcal{S}$. Since

$$
h\left(1 \otimes \alpha, \mathfrak{N}_{Z} \otimes P \mid \mathfrak{E}\right)=\lim _{n \rightarrow \infty} h\left(\mathcal{S}_{n}, 1 \otimes \alpha, \mathfrak{N}_{Z} \otimes P \mid \mathfrak{E}\right),
$$

we deduce from (3-1) that

$$
\begin{aligned}
h\left(\mathcal{S}_{n}, 1 \otimes \alpha, \mathfrak{N}_{Z} \otimes P \mid \mathfrak{E}\right) & =\int_{A_{n} \times X} H\left(\bigvee_{i=0}^{2^{n}-1} \alpha\left(x, \gamma^{i} x\right) P_{\gamma^{i} x} \mid \mathfrak{E}\right) d \mu(z) d \mu(x) \\
& =\int_{X} \frac{1}{2^{n}} H\left(\bigvee_{i=0}^{2^{n}-1} \alpha\left(x, \gamma^{i} x\right) P_{\gamma^{i} x} \mid \mathfrak{E}\right) d \mu(x) \\
& =\frac{1}{2^{n}} H\left(\bigvee_{i=0}^{2^{n}-1} \gamma_{\alpha}^{-i} P \mid \mathfrak{B}_{X} \otimes \mathfrak{E}\right)
\end{aligned}
$$

Hence $h\left(1 \otimes \alpha, \mathfrak{N}_{Z} \otimes P \mid \mathfrak{E}\right)=h\left(\gamma_{\alpha}, P \mid \mathfrak{B}_{X} \otimes \mathfrak{E}\right)$. 
Corollary 3.7. $h(\alpha \mid \mathfrak{E})=h\left(\gamma_{\alpha} \mid \mathfrak{B}_{X} \otimes \mathfrak{E}\right)$ and $\Pi(\alpha \mid \mathfrak{E})=\Pi\left(\gamma_{\alpha} \mid \mathfrak{B}_{X} \otimes \mathfrak{E}\right)$. Hence $\alpha$ is $\mathfrak{E}$-relatively $C P E$ if and only if $\gamma_{\alpha}$ is $\left(\mathfrak{B}_{X} \otimes \mathfrak{E}\right)$-relatively CPE.

The above two statements (3.6 and 3.7) will be extended later in the general setup of amenable group actions.

Recall a concept from [RW]. Given two finite subsets $K$ and $F$ of $G$, we say that $F$ is $K$-spread if $g_{1} g_{2}^{-1} \notin K$ for any $g_{1} \neq g_{2} \in F$.

Theorem 3.8 (cf. [RW, Theorem 2.12]). Let $\alpha$ be $\mathfrak{E}$-relatively CPE. Given $\epsilon>0$, then there exists a finite subset $K$ in $G$ such that for each finite $K$-spread subset $F \subset G$,

$$
\left\|\frac{1}{\# F} H\left(\bigvee_{g \in F} \alpha(x, g x) P_{g x} \mid \mathfrak{E}\right)-\frac{1}{\# F} \sum_{g \in F} H\left(P_{g x} \mid \mathfrak{E}\right)\right\|_{1}<\epsilon
$$

Proof. Let $\gamma$ be a generator of $\mathcal{R}$. Since $\# \mathcal{R}(x)=\infty$ a.e., $\gamma$ acts freely. Given $x \in X$, we define a linear order $\geq_{x}$ on $\mathcal{R}(x)$ by setting $v \geq_{x} w$ if $v=\gamma^{n} w$ for some $n \geq 0$. By Corollary 3.7, $\gamma_{\alpha}$ is $\left(\mathfrak{B}_{X} \otimes \mathfrak{E}\right)$-relatively CPE. Since $\bigcap_{m} \bigvee_{i>m} \gamma_{\alpha}{ }^{-i} P \subset$ $\Pi\left(\gamma_{\alpha}\right)$ (see $\left.[\mathrm{RoS}]\right)$ and $\Pi\left(\gamma_{\alpha}\right) \subset \Pi\left(\gamma_{\alpha} \mid \mathfrak{B}_{X} \otimes \mathfrak{E}\right)=\mathfrak{B}_{X} \otimes \mathfrak{E}$, it follows that

$$
\bigcap_{m}\left(\bigvee_{i>m} \gamma_{\alpha}{ }^{-i} P \vee\left(\mathfrak{B}_{X} \otimes \mathfrak{E}\right)\right)=\mathfrak{B}_{X} \otimes \mathfrak{E}
$$

and therefore $H\left(P_{x} \mid \bigvee_{i>m} \alpha\left(x, \gamma^{i} x\right) P_{\gamma^{i} x} \vee \mathfrak{E}\right) \rightarrow H\left(P_{x} \mid \mathfrak{E}\right)$ a.e. as $m \rightarrow \infty$. Hence for some $n>0$ there is a subset $B, \mu(B)>1-\epsilon^{2} / 2$ with

$$
H\left(P_{x} \mid \bigvee_{x^{\prime} \geq x \gamma^{n} x} \alpha\left(x, x^{\prime}\right) P_{x^{\prime}} \vee \mathfrak{E}\right)>H\left(P_{x} \mid \mathfrak{E}\right)-\epsilon \log (\# P) \quad \text { for all } x \in B
$$

Choose $K \in \operatorname{Fin}(G)$ "large" so that $\left\{\gamma x, \ldots, \gamma^{n} x\right\} \in K x$ for all $x$ from a subset $A \subset X$ with $\mu(A)>1-\epsilon^{2} / 2$.

Now given a $K$-spread subset $F \in \operatorname{Fin}(G)$, we furnish $F \times X$ with the product of the counting measure on $F$ and $\mu$ on $X$. Then the measure of the subset

$$
\{(g, x) \in F \times X \mid g x \in A \cap B\}=\bigcup_{g \in F}\{g\} \times g^{-1}(A \cap B)
$$

is greater than $\left(1-\epsilon^{2}\right) \# F$. From this and Fubini theorem we deduce that there is a subset $C \subset X, \mu(C)>1-\epsilon$, and a measurable map $C \ni x \mapsto F_{x} \subset F$ with $\# F_{x}>$ $(1-\epsilon) \# F$ and $F_{x} x \subset A \cap B$ for all $x \in C$. Since $F$ (and hence $F_{x}$ ) is $K$-spread, for any two (distinct) points $v$ and $w$ of $F_{x} x$ we have $w \notin K v \supset\left\{\gamma v, \ldots, \gamma^{n} v\right\}$. Thus 
if $w \gtrless_{x} v$ then $w \gtrless_{x} \gamma^{n} v$. Hence for $x \in C$,

$$
\begin{aligned}
H\left(\bigvee_{g \in F} \alpha(x, g x) P_{g x} \mid \mathfrak{E}\right) & \geq H\left(\bigvee_{x^{\prime} \in F_{x} x} \alpha\left(x, x^{\prime}\right) P_{x^{\prime}} \mid \mathfrak{E}\right) \\
& =\sum_{x^{\prime} \in F_{x} x} H\left(\alpha\left(x, x^{\prime}\right) P_{x^{\prime}} \mid \bigvee_{F_{x} x \ni z \gtrless_{x} x^{\prime}} \alpha(x, z) P_{z} \vee \mathfrak{E}\right) \\
& \geq \sum_{x^{\prime} \in F_{x} x} H\left(P_{x^{\prime}} \mid \bigvee_{z \gtrless_{x} \gamma^{n} x^{\prime}} \alpha\left(x^{\prime}, z\right) P_{z} \vee \mathfrak{E}\right) \\
& \geq \sum_{x^{\prime} \in F_{x} x}\left(H\left(P_{x^{\prime}} \mid \mathfrak{E}\right)-\epsilon \log (\# P)\right) \\
& \geq \sum_{x^{\prime} \in F x} H\left(P_{x^{\prime}} \mid \mathfrak{E}\right)-\left(\epsilon \# F+\#\left(F \backslash F_{x}\right)\right) \log (\# P) \\
& \geq \sum_{g \in F} H\left(P_{g x} \mid \mathfrak{E}\right)-\# F \cdot 2 \epsilon \log (\# P)
\end{aligned}
$$

On the other hand, $H\left(\bigvee_{g \in F} \alpha(x, g x) P_{g x} \mid \mathfrak{E}\right) \leq \sum_{g \in F} H\left(P_{g x} \mid \mathfrak{E}\right)$ for each $x \in X$, and we conclude immediately that

$$
\begin{aligned}
\int_{X} \mid \frac{1}{\# F} H\left(\bigvee_{g \in F} \alpha(x, g x) P_{g x} \mid \mathfrak{E}\right) & -\frac{1}{\# F} \sum_{g \in F} H\left(P_{g x} \mid \mathfrak{E}\right) \mid d \mu(x) \\
& <\int_{X \backslash C} 2 \log (\# P) d \mu+2 \epsilon \log (\# P) \leq 4 \epsilon \log (\# P) .
\end{aligned}
$$

This is equivalent to the statement of the theorem.

Proof of Theorem 0.1. Let us assume that $\mathcal{R}$ is generated by a Bernoullian $G$-action. Then $\mathcal{R}$ is ergodic, $G$ acts freely and its centralizer is ergodic. We define a cocycle $\beta_{T}: \mathcal{R} \rightarrow \operatorname{Aut}_{\mathfrak{E}}(Y, \nu)$ like in Definition 3.1. By Corollary 3.4, $\beta_{T}$ is E-relatively CPE. It remains to apply Theorem 3.8 with $\alpha:=\beta_{T}$ and $P:=\mathfrak{N}_{X} \otimes Q$.

\section{Abramov-Rokhlin Entropy addition Formula}

Let $t \in \operatorname{Aut}\left(X, \mathfrak{B}_{X}, \mu\right)$. Given two $t$-factors $\mathfrak{A} \subset \mathfrak{F}$ and two finite $X$-partitions $P$ and $Q$, it follows from the classical Pinsker formula that

$$
h(t, P \vee Q \mid \mathfrak{A})=h(t, Q \mid \mathfrak{A})+h\left(t, P \mid \mathfrak{A} \vee Q^{\infty}\right),
$$

where $Q^{\infty}=\bigvee_{i \in \mathbb{Z}} t^{i} Q$. First taking the supremum over all $\mathfrak{F}$-measurable $Q$ and then over all arbitrary $P$, we obtain

$$
h(t \mid \mathfrak{A})=h((t \uparrow \mathfrak{F}) \mid \mathfrak{A})+h(t \mid \mathfrak{F}) .
$$

Proof of Theorem 0.2. Let $\mathcal{R}$ be generated by a free $G$-action and a transformation $\gamma$ stand for an-aperiodic-generator of $\mathcal{R}$. We define two cocycles $\alpha: \mathcal{R} \rightarrow$ $\operatorname{Aut}(Y, \nu \uparrow \mathfrak{E})$ and $\beta_{T}: \mathcal{R} \rightarrow \operatorname{Aut}(Y, \nu)$ by setting

$$
\alpha(g x, x)=T_{g} \uparrow \mathfrak{E}, \quad \beta_{T}(g x, x)=T_{g}
$$


for all $x \in X, g \in G$. It follows from Theorems 3.3(ii), 3.6 and (4-1) that

$$
\begin{aligned}
h(T) & =h\left(\beta_{T}\right)=h\left(\gamma_{\beta_{T}} \mid \mathfrak{B}_{X} \otimes \mathfrak{N}_{Y}\right) \\
& =h\left(\left(\gamma_{\beta_{T}} \mid \mathfrak{B}_{X} \otimes \mathfrak{E}\right) \mid \mathfrak{B}_{X} \otimes \mathfrak{N}_{Y}\right)+h\left(\gamma_{\beta_{T}} \mid \mathfrak{B}_{X} \otimes \mathfrak{E}\right) \\
& =h\left(\gamma_{\alpha} \mid \mathfrak{B}_{X} \otimes \mathfrak{N}_{Y}\right)+h\left(\gamma_{\beta_{T}} \mid \mathfrak{B}_{X} \otimes \mathfrak{E}\right) \\
& =h\left(\alpha \mid \mathfrak{B}_{X} \otimes \mathfrak{N}_{Y}\right)+h\left(\beta_{T} \mid \mathfrak{B}_{X} \otimes \mathfrak{E}\right) \\
& =h(T\lceil\mathfrak{E})+h(T \mid \mathfrak{E}) . \quad
\end{aligned}
$$

\section{FACTOR ORBIT EQUIVALENT ACTIONS HaVE THE SAME RELATIVE ENTROPY}

In this section we prove Theorem 0.3. Moreover, as promised, we extend Theorem 3.6 and Corollary 3.7 to actions of arbitrary countable amenable groups.

Let $\left(Z, \mathfrak{B}_{Z}, \kappa\right)$ be a standard probability space and $\mathfrak{F}$ a sub- $\sigma$-algebra of $\mathfrak{B}_{Z}$.

Theorem 5.1. Let $T$ be an action of $G$ on $(Y, \nu)$ and $\beta$ a cocycle of the T-orbit equivalence relation with values in $A u t_{\widetilde{F}}(Z, \kappa)$. Then

$$
h(\beta, P \mid \mathfrak{F})=h\left(T^{\beta}, P \mid \mathfrak{B}_{Y} \otimes \mathfrak{F}\right)
$$

for each finite partition $P$ of $Y \times Z$.

Proof. Assume that $\mathcal{R}$ is the orbit equivalence relation of a free $G$-action like in $\S 3$, 4. We define three cocycles $\beta_{T}: \mathcal{R} \rightarrow \operatorname{Aut}(Y, \nu), \beta^{\prime}: \mathcal{R} \rightarrow \operatorname{Aut}(Y \times Z, \nu \times \kappa)$ and $1 \otimes \beta: \mathcal{R}(\alpha) \rightarrow \operatorname{Aut}(Z, \kappa)$ by setting

$$
\beta_{T}(g x, x)=T_{g}, \quad \beta^{\prime}(g x, x)=\left(T_{g}\right)_{\beta}, \quad(1 \otimes \beta)\left(\left(g x, T_{g} y\right),(x, y)\right)=\beta\left(T_{g} y, y\right)
$$

for all $x \in X, y \in Y, g \in G$. Let $\gamma$ be a generator of $\mathcal{R}$ with $h(\gamma)<\infty$. It is routine to verify that $\gamma_{\beta^{\prime}}=\left(\gamma_{\beta_{T}}\right)_{1 \otimes \beta}$. We deduce from Theorems 3.3, 3.6 that

$$
\begin{aligned}
h\left(T^{\beta}, P \mid \mathfrak{B}_{Y} \otimes \mathfrak{F}\right) & =h\left(\beta^{\prime}, \mathfrak{N}_{X} \otimes P \mid \mathfrak{B}_{Y} \otimes \mathfrak{F}\right) \\
& =h\left(\gamma_{\beta^{\prime}}, \mathfrak{N}_{X} \otimes P \mid \mathfrak{B}_{X} \otimes \mathfrak{B}_{Y} \otimes \mathfrak{F}\right) \\
& =h\left(\left(\gamma_{\beta_{T}}\right)_{1 \otimes \beta}, \mathfrak{N}_{X} \otimes P \mid \mathfrak{B}_{X \times Y} \otimes \mathfrak{F}\right) \\
& =h\left(1 \otimes \beta, \mathfrak{N}_{X} \otimes P \mid \mathfrak{F}\right)=h(\beta, P \mid \mathfrak{F}) .
\end{aligned}
$$

Corollary 5.2. $h(\beta \mid \mathfrak{F})=h\left(T^{\beta} \mid \mathfrak{B}_{Y} \otimes \mathfrak{F}\right)$ and $\Pi(\beta \mid \mathfrak{F})=\Pi\left(T^{\beta} \mid \mathfrak{B}_{Y} \otimes \mathfrak{F}\right)$. Hence $\beta$ is $\mathfrak{F}$-relatively $C P E$ if and only if $T^{\beta}$ is $\left(\mathfrak{B}_{Y} \otimes \mathfrak{F}\right)$-relatively CPE.

From this and Theorems 0.2 and 2.13 we deduce

Corollary 5.3. If $\beta$ is recurrent then $h\left(T^{\beta}\right)=h(T)$.

Corollary 5.4. Let $T$ and $U$ be actions of countable amenable groups $G$ and $F$ respectively on $(Y, \nu)$. Suppose that they have the same orbits, i.e. generate the same equivalence relation, say $\mathcal{S}$. Then for each cocycle $\beta: \mathcal{S} \rightarrow \operatorname{Aut}_{\mathfrak{F}}(Z, \kappa)$ and a finite partition $P$ of $Y \times Z$, we have

$$
h\left(T^{\beta}, P \mid \mathfrak{B}_{Y} \otimes \mathfrak{F}\right)=h\left(U^{\beta}, P \mid \mathfrak{B}_{Y} \otimes \mathfrak{F}\right),
$$

where $T^{\beta}$ and $U^{\beta}$ are the $\beta$-skew product extensions of $T$ and $U$ respectively.

Proof of Theorem 0.3. Since $\mathfrak{E}$ is class-bijective, each of the actions $T$ and $U$ is isomorphic to a skew-product extension of the factor-action. Moreover, since $T$ and $U$ are $\mathfrak{E}$-orbit equivalent, the factor-actions have the same orbits and the corresponding extending cocycles are identical. Hence we may apply Corollary 5.4 (with $\mathfrak{F}$ trivial). 


\section{Relative independence, Pinsker algebras and \\ ENTROPY ADDITION FORMULA FOR AMENABLE ACTIONS}

Our purpose here is to prove Theorem 0.4.

Lemma 6.1. Let $\gamma$ be a transformation of $\left(X, \mathfrak{B}_{X}, \mu\right)$ and $\mathfrak{B}_{1}, \mathfrak{B}_{2}, \mathfrak{F}$ three factors of $\gamma$ with $\mathfrak{F} \subset \mathfrak{B}_{1} \cap \mathfrak{B}_{2}$.

(i) If $\gamma \uparrow \mathfrak{B}_{1}$ is $\mathfrak{F}$-relatively $C P E$ and $h\left(\gamma \uparrow \mathfrak{B}_{2} \mid \mathfrak{F}\right)=0$ then $\mathfrak{B}_{1}$ and $\mathfrak{B}_{2}$ are $\mathfrak{F}$-relatively independent.

(ii) If $\gamma \uparrow \mathfrak{B}_{1}$ is $\mathfrak{F}$-relatively CPE then $\gamma \uparrow\left(\mathfrak{B}_{1} \vee \mathfrak{B}_{2}\right)$ is $\mathfrak{B}_{2}$-relatively CPE.

(iii) If $\mathfrak{B}_{1}$ and $\mathfrak{B}_{2}$ are $\mathfrak{F}$-relatively independent then $\Pi\left(\gamma \uparrow\left(\mathfrak{B}_{1} \vee \mathfrak{B}_{2}\right) \mid \mathfrak{F}\right)=$ $\Pi\left(\gamma \uparrow \mathfrak{B}_{1} \mid \mathfrak{F}\right) \vee \Pi\left(\gamma \uparrow \mathfrak{B}_{2} \mid \mathfrak{F}\right)$.

(iv) $\mathfrak{B}_{1}$ and $\mathfrak{B}_{2}$ are $\mathfrak{F}$-relatively independent if and only if the Pinsker factors $\Pi\left(\gamma\left\lceil\mathfrak{B}_{1} \mid \mathfrak{F}\right)\right.$ and $\Pi\left(\gamma\left\lceil\mathfrak{B}_{2} \mid \mathfrak{F}\right)\right.$ are $\mathfrak{F}$-relatively independent and

$$
h\left(\gamma \uparrow\left(\mathfrak{B}_{1} \vee \mathfrak{B}_{2}\right) \mid \mathfrak{F}\right)=h\left(\gamma \uparrow \mathfrak{B}_{1} \mid \mathfrak{F}\right)+h\left(\gamma \uparrow \mathfrak{B}_{2} \mid \mathfrak{F}\right) .
$$

Proof. (i) This is a relative version of the disjointness theorem of Pinsker [Ro, $\S 13.2]$. It can be demonstrated in the same way as there. We remark also that (i) follows from (iv).

(ii) and (iii) follows easily from the relative Rokhlin-Sinai theorem about equivalence of the CPE-property and the $K$-property.

(iv) An absolute version - for $\mathfrak{F}$ trivial — was proved in [Be]. The relative version can be demonstrated in a similar way. Remark that the ergodicity assumption in [Be] is not essential and can be omitted.

Proof of Theorem 0.4. Let $G \times X \ni(g, x) \mapsto g x \in X$ be a Bernoullian action of $G$ on $(X, \mu)$ and $\mathcal{R}$ its orbit equivalence relation. We let $\mathfrak{A}_{3}=\mathfrak{A}_{1} \vee \mathfrak{A}_{2}$ and define four cocycles $\beta_{T}, \beta_{1}, \beta_{2}, \beta_{3}$ of $\mathcal{R}$ by setting

$$
\beta_{T}(g x, x)=T_{g}, \quad \beta_{i}(g x, x)=T_{g} \uparrow \mathfrak{A}_{i}, \quad i=1, \ldots, 3 .
$$

Let a transformation $\gamma$ generate $\mathcal{R}$. It is clear that $\gamma_{\beta_{i}}=\gamma_{\beta_{T}} \uparrow\left(\mathfrak{B}_{X} \otimes \mathfrak{A}_{i}\right)$, $i=1, \ldots, 3$.

(i) By Corollary 3.4, Theorem 3.3(ii) and the assumptions of the theorem, $\beta_{1}$ is $\mathfrak{E}$-relatively $\mathrm{CPE}$ and $h\left(\beta_{2} \mid \mathfrak{E}\right)=0$. It follows from Corollary 3.7 that the transformation $\gamma_{\beta_{1}}$ is $\left(\mathfrak{B}_{X} \otimes \mathfrak{E}\right)$-relatively $\mathrm{CPE}$ and $h\left(\gamma_{\beta_{2}} \mid \mathfrak{B}_{X} \otimes \mathfrak{E}\right)=0$. We deduce from Lemma $6.1(\mathrm{i})$ that $\mathfrak{B}_{X} \otimes \mathfrak{A}_{1}$ and $\mathfrak{B}_{X} \otimes \mathfrak{A}_{2}$ are $\left(\mathfrak{B}_{X} \otimes \mathfrak{E}\right)$-relatively independent. It follows that $\mathfrak{A}_{1}$ and $\mathfrak{A}_{2}$ are $\mathfrak{E}$-relatively independent. Remark also that (i) follows from (iv).

(ii) Since $\gamma_{\beta_{1}}$ is $\mathfrak{B}_{X} \otimes \mathfrak{E}$-relatively CPE, we deduce from Lemma 6.1(ii) that $\gamma_{\beta_{3}}$ is $\left(\mathfrak{B}_{X} \otimes \mathfrak{A}_{2}\right)$-relatively CPE. It follows from Corollaries 3.4 and 3.7 that $T \uparrow \mathfrak{A}_{3}$ is $\mathfrak{A}_{2}$-relatively CPE.

(iii) We deduce from Corollaries 3.4, 3.7 and Lemma 6.1(iii) that

$$
\begin{aligned}
\mathfrak{B}_{X} \otimes \Pi\left(T \uparrow\left(\mathfrak{A}_{1} \vee \mathfrak{A}_{2}\right) \mid \mathfrak{E}\right) & =\Pi\left(\gamma_{\beta_{3}} \mid \mathfrak{B}_{X} \otimes \mathfrak{E}\right) \\
& =\Pi\left(\gamma_{\beta_{1}} \mid \mathfrak{B}_{X} \otimes \mathfrak{E}\right) \vee \Pi\left(\gamma_{\beta_{2}} \mid \mathfrak{B}_{X} \otimes \mathfrak{E}\right) \\
& =\left(\mathfrak{B}_{X} \otimes \Pi\left(T \uparrow \mathfrak{A}_{1} \mid \mathfrak{E}\right)\right) \vee\left(\mathfrak{B}_{X} \otimes \Pi\left(T \uparrow \mathfrak{A}_{2} \mid \mathfrak{E}\right)\right) \\
& =\mathfrak{B}_{X} \otimes\left(\Pi\left(T \uparrow \mathfrak{A}_{1} \mid \mathfrak{E}\right) \vee \Pi\left(T \uparrow \mathfrak{A}_{2} \mid \mathfrak{E}\right)\right)
\end{aligned}
$$

(iv) is proved via the same trick with $3.4,3.7$ and 6.1 (iv). 
Appendix: Proof of Theorem 1.1

We define a function $f: \operatorname{Fin}(G) \rightarrow \mathbb{R}$ by setting $f(A)=H\left(\bigvee_{a \in A} T_{a}^{-1} P \mid \mathfrak{E}\right)$ Clearly,

$$
\begin{aligned}
& f(A g)=f(A) \\
& f(A \cup B)+f(A \cap B) \leq f(A)+f(B), \\
& f(A) \leq \# A \cdot H(P \mid \mathfrak{E})
\end{aligned}
$$

for all $A, B \in \operatorname{Fin}(G)$ and $g \in G$. It is enough to prove that

$$
\varlimsup_{\Phi} \frac{f(A)}{\# A}=h(T, P \mid \mathfrak{E}) .
$$

The inequality " $\geq$ " in (A-4) is obvious. To prove " $\leq$ " we demonstrate first that $f$ is subadditive, i.e. if $1_{A}=\sum_{j} \alpha_{j} 1_{A_{j}}$ for some $A, A_{j} \in \operatorname{Fin}(G)$ and positive $\alpha_{j}$ then

$$
f(A) \leq \sum_{j} \alpha_{j} f\left(A_{j}\right)
$$

Enumerate the atoms of the partition of $A$ generated by $\left(A_{j}\right)_{j}$ and denote by $K_{i}$ the union of the first $i$ atoms. Then

$$
\emptyset=K_{0} \subsetneq K_{1} \subsetneq \cdots \subsetneq K_{n}=A
$$

for some $n \in \mathbb{N}$. Moreover, it is easy to see that if $\left(K_{i} \backslash K_{i-1}\right) \cap A_{j} \neq \emptyset$ for some $i, j$ then $K_{i}=K_{i-1} \cup\left(A_{j} \cap K_{i}\right)$. It follows from this and (A2) that

$$
f\left(K_{i}\right)-f\left(K_{i-1}\right) \leq f\left(K_{i} \cap A_{j}\right)-f\left(K_{i-1} \cap A_{j}\right) .
$$

Select elements $k_{i} \in K_{i} \backslash K_{i-1}, i=1, \ldots, n$. Then

$$
\begin{aligned}
f(A) & =\sum_{i=1}^{n}\left(f\left(K_{i}\right)-f\left(K_{i-1}\right)\right) \\
& =\sum_{i=1}^{n}\left(\sum_{j} \alpha_{j} 1_{A_{j}}\left(k_{i}\right)\right)\left(f\left(K_{i}\right)-f\left(K_{i-1}\right)\right) \\
& =\sum_{j} \alpha_{j} \sum_{i=1}^{n} 1_{A_{j}}\left(k_{i}\right)\left(f\left(K_{i}\right)-f\left(K_{i-1}\right)\right) \\
& \stackrel{(\mathrm{A}-6)}{\leq} \sum_{j} \alpha_{j} \sum_{i=1}^{n} 1_{A_{j}}\left(k_{i}\right)\left(f\left(K_{i} \cap A_{j}\right)-f\left(K_{i-1} \cap A_{j}\right)\right) \\
& =\sum_{j} \alpha_{j} f\left(A_{j}\right),
\end{aligned}
$$

as desired. 
Next, given $\epsilon>0$, we select $B \in \operatorname{Fin}(G)$ such that $\frac{f(B)}{\# B}<h(T, P \mid \mathfrak{E})+\epsilon$. In view of (A-1) we may assume without loss of generality that $B \ni 1_{G}$. Take any $A \in \Phi[B, \epsilon]$. Since $1_{A}=\frac{1}{\# B} \sum_{B g \cap A \neq \emptyset} 1_{B g \cap A}$, we have

$$
\begin{aligned}
f(A) & \stackrel{(\mathrm{A}-5)}{\leq} \frac{1}{\# B} \sum_{B g \cap A \neq \emptyset} f(B g \cap A) \\
& =\frac{1}{\# B} \sum_{B g \subset A} f(B g \cap A)+\frac{1}{\# B} \sum_{\substack{B g \cap A \neq \emptyset, B g \not \subset A}} f(B g \cap A) \\
& \stackrel{(\mathrm{A}-1)}{\leq} \#\{g \in A \mid B g \subset A\} f(B)+\#\{g \mid B g \cap A \neq \emptyset, B g \not \subset A\} H(P \mid \mathfrak{E}) .
\end{aligned}
$$

Hence

$$
\frac{f(A)}{\# A} \leq(1-\epsilon) f(B)+\epsilon \# B \cdot H(P \mid \mathfrak{E}) \leq(1-\epsilon)(h(T, P \mid \mathfrak{E})+\epsilon)+\epsilon \# B \cdot H(P \mid \mathfrak{E})
$$

This implies $\varlimsup_{\Phi} \frac{f(A)}{\# A} \leq h(T, P \mid \mathfrak{E})$.

\section{REFERENCES}

[Be] K. R. Berg, Independence and additive entropy, Proc. Amer. Math. Soc. 51 (1975), 366370.

[CFW] A. Connes, J. Feldman, and B. Weiss, An amenable equivalence relation is generated by a single transformation, Ergod. Th. \& Dynam. Sys. 1 (1981), 431-450.

[Da1] A. I. Danilenko, Comparison of cocycles of measured equivalence relations and lifting problems, Ergod. Th. \& Dynam. Sys. 18 (1998), 125-151.

[Da2] , Quasinormal subrelations of ergodic equivalence relations, Proc. Amer. Math. Soc. 126 (1998), 3361-3370.

[Da3] _ Endomorphisms of measured equivalence relations, cocycles with values in nonlocally compact groups and applications, Ergod. Th. \& Dynam. Sys. 19 (1999), 571-590.

[DaG] A. I. Danilenko and V. Ya. Golodets, Extension of cocycles to normalizer elements, outer conjugacy and related problems, Trans. Amer. Math. Soc. 348 (1996), 4857-4882.

[Dy] H. A. Dye, On groups of measure preserving transformations. I, Amer. J. Math. 81 (1959), 119-159.

[FM] J. Feldman and C. C. Moore, Ergodic equivalence relations, cohomology, and von Neumann algebras. I, Trans. Amer. Math. Soc. 234 (1977), 289-324.

[FSZ] J. Feldman, C. E. Sutherland, and R. J. Zimmer, Subrelations of ergodic equivalence relations, Ergod. Th. and Dynam. Syst. 9 (1989), 239-269.

[GTW] E. Glasner, J.-P. Thouvenot and B. Weiss, Entropy theory without past, Erg. Th. \& Dynam. Sys. (to appear).

[GoS] V. Ya. Golodets and S. D. Sinelshchikov, Classification and structure of cocycles of amenable ergodic equivalence relation, J. Funct. Anal. 121 (1994), 455-485.

[Ki] J. C. Kieffer, A generalized Shannon-McMillan theorem for the action of an amenable group on a probability space, Ann. Prob. 3 (1975), 1031-1037.

[Ol] J. M. Ollagnier, Ergodic theory and statistical mechanics, Lecture Notes in Math., 1115, Springer-Verlag, Berlin, Heidelberg, New York, 1985.

[OW] D. S. Ornstein and B. Weiss, Entropy and isomorphism theorems for actions of amenable groups, J. d'Analyse Math. 48 (1987), 1-141.

[Ro] V. A. Rokhlin, Lectures on the entropy theory for transformations with invariant measure, Uspekhi Mat. Nauk 22 (1967), 4-56. (in Russian)

[RoS] V. A. Rokhlin and Ya. G. Sinai, Construction and properties of invariant measurable partitions, Dokl. Acad. Sci. USSR 141 (1961), 1038-1041. (in Russian) 
[RW] D. J. Rudolph and B. Weiss, Entropy and mixing for amenable group actions, Annals of Math. (to appear)

[Sc] K. Schmidt, Algebraic ideas in ergodic theory, CBMS Regional Conf. Ser. in Math., No 76, Amer. Math. Soc., Providence, RI, 1990.

[WZ] T. Ward and Q. Zhang, The Abramov-Rokhlin entropy addition formula for amenable group actions, Mh. Math. 114 (1992), 317-329.

[Zi] R. Zimmer, Hyperfinite factors and amenable group actions, Invent. Math. 41 (1877), 23-31.

Department of Mathematics, College of Natural Science, Ajou University, SuWON 442-749, KOREA

E-mail address: danilenk@madang.ajou.ac.kr 